\title{
On 'spot' evolution under an adverse pressure gradient
}

\author{
By F. T. SMITH AND S. N. TIMOSHIN \\ Department of Mathematics, University College London, \\ Gower Street, London WC1E 6BT, UK
}

(Received 9 June 1999 and in revised form 11 September 2000)

The unsteady travelling 'spots' or spot-like disturbances are produced, in an otherwise planar boundary layer, by an initial impulse/blip, from wall forcing or from nearby external forcing. Theory and computations are described for the evolving spot-like structure, yielding initial-value problems for inviscid spot-like disturbances, commencing near the onset of an adverse pressure gradient. A transient stage incorporates the initial conditions, following which adverse pressure gradient effects become significant. Leading and trailing critical layers then form, which confine and define the spot-like disturbance, and these depart from the wall downstream accompanied by disturbance amplification and mean flow distortion. The interplay of adverse pressure gradient effects with three-dimensionality, nonlinearity and non-parallelism is considered in turn.

Three-dimensional effects provoke a universal closed planform of spot-like disturbance, which has a different side behaviour from the zero-gradient case. Nonlinear interactions eventually change the internal structure, particularly at the spot-like disturbance leading edge, while pointing to the mean-flow alteration underhanging the spot-like disturbance and to a pressure-feedback alteration for the region behind the spot-like disturbance. These two alterations offer complementary mechanisms for describing the calmed region trailing a spot-like disturbance, in which an attached thinned wall layer is identified. Non-parallel effects lead to enhanced spot-like disturbance growth and larger-scale/shorter-scale interactive behaviour downstream. The approach to separation is also considered, yielding maximal growth for small spot-like disturbances at $5 / 6$ of the way from the minimum pressure position to the separation position. Links with recent experiments on adverse-gradient spot-like disturbances and with findings on calmed region properties are investigated, as well as the unsteady forcing effects from an incident relatively thick vortical wake outside the boundary layer.

\section{Introduction}

The creation of so-called 'spots' of unsteady (spatially confined) transitional or turbulent disturbed motion within an otherwise steady boundary layer is of much concern in engine flow design and elsewhere. Here, the term 'spot' is used to denote a spot-like disturbance or, in two dimensions, a strip. 'Spot' propagation models are used in turbomachinery transition predictions (as described by Abu Ghannam \& Shaw 1980; Gostelow et al. 1993; Clarke, Jones \& LaGraff 1994; Narasimha 1997; Johnson 1997; for instance) but are also relevant to other contexts with deep-transition physics (for example Smith \& Bowles 1992; Li et al. 1998; Savin, Smith \& Allen 1999). Most of the many 'spot' experiments are for basic boundary layers under nominally zero pressure gradient, as discussed in the reviews in the references above. 
Gostelow and colleagues $(1993,1994)$ recently led the study of the effects of adverse pressure gradients, which are observed to provoke much larger spreading rates of the 'spot' planform and quite distinct off-centre effects, among many other important new features which have to be taken into account compared with zero or favourable gradient cases. See also Seifert \& Wygnanski (1995), Seifert (1997), Gostelow et al. (1997), van Hest (1997) and Howell (1998). Computationally, direct simulations have yielded relatively little on adverse gradient effects, especially concerning their physical understanding, but empirically based modelling has been performed by Johnson (1997, 1998), Solomon, Walker \& Gostelow (1996), Johnson \& Ercan (1996) and Steelant \& Dick (1996). Little basic theoretical nonlinear study without empiricism has been made for 'spots' until quite recently: see references later in this section. Any such study was without significant adverse pressure gradients.

On compressor and turbine blades there are substantial areas of adverse pressure gradient, owing to high loading. These areas are commonly observed to leave enhanced calmed regions trailing behind any travelling 'spot' disturbance (see Gostelow et al. 1997), the calmed regions being of practical importance in the production of extensive patches of laminar or relaminarized flow on the blades and being associated in practice (observations by Gostelow, Hodson, Jones, Seifert) with the production of relatively thin attached layers near the wall behind the 'spot'.

The lack of, and need for, deep physical understanding of 'spots' was highlighted at the 1997 Minnowbrook workshop on transition in turbomachinery (Narasimha 1997). Stress was placed on the unknown roles, during 'spot' evolution, of adverse pressure gradients, of calmed regions, of separations, of high free-stream turbulence, and on the need for more basic theoretical study. Major issues in 'spot' dynamics concern: the determination of the sharp edges and global shape of the typical 'spots'; the underlying reason for the calmed region, with its advantageous relaminarizing and stabilizing effect; pressure-gradient influences; and the breakdown to shorter lengthscales inside the 'spot'. Some of these issues are tackled here, hopefully to help resolve the fundamental flow structure(s), advance physical understanding and quantify the influences of pressure gradients. Again, most flows of real interest have large characteristic Reynolds numbers.

Given the amount of interest in 'spots', the present paper addresses their scales, flow structure and controlling parameters, under adverse pressure gradients. The 'spot' is initiated here by introduction of a three-dimensional unsteady disturbance into a laminar planar boundary layer (on a blade, airfoil or internal wall), by means of an abrupt kick (pulse, blip), such as in the pulsed wall membrane experiments by Grass (private communications 1992-95) or the wall injection/suction experiments by Seifert, Gostelow and their colleagues (private communications 1997-99) and by Howell (1998) and Seifert \& Hodson (2000). 'Spots' in reality are produced in many different ways, by forcing at the wall as above, or external to the boundary layer as addressed in the Appendix, or both. The emphasis then in this work is on the free evolution problem for a general initial disturbance, as distinct from a fixed frequency or fixed wavelength disturbance. The 'spots' of concern are rotational, distorting the shear of the original decelerating boundary layer three-dimensionally, and are predominantly inviscid apart from their eventual wall-sublayer breakdown. All transients and growing amplitude components of the travelling 'spot' flow must be included.

A crucial position for the initiation of the 'spot' is near the point of minimum basic pressure, at the onset of the adverse pressure gradient. Inflectional instability enters there via a near-wall sublayer. Gostelow's experimental 'spots', in particular, 


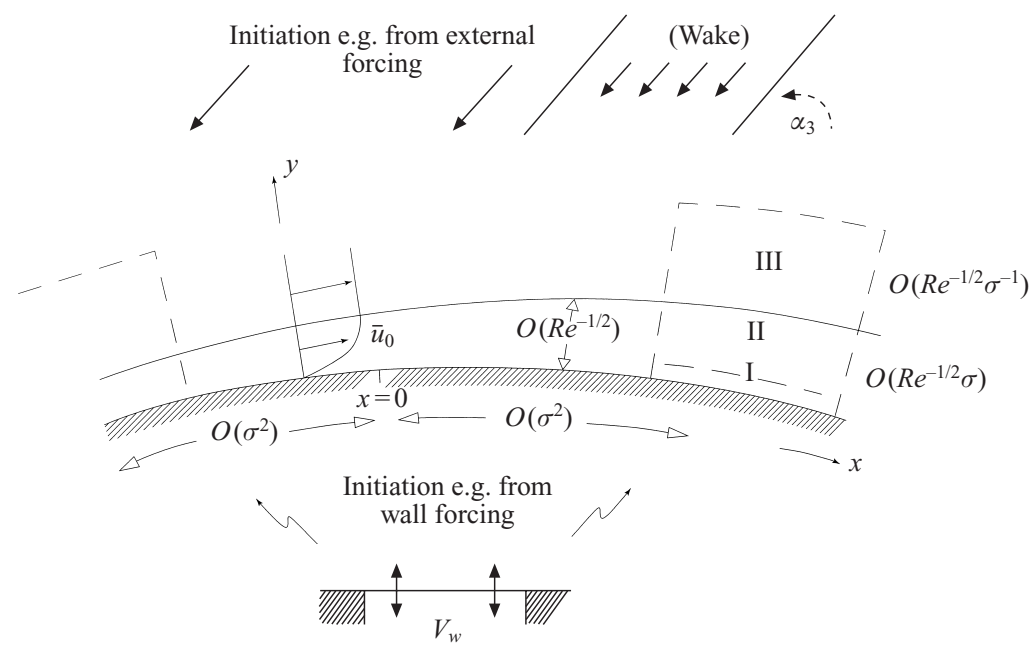

FIGURE 1. The flow structure and scales, near the onset of the adverse pressure gradient: see $\S 2$. The disturbances of $\S \S 3$ onwards start typically at a distance $O\left(\sigma^{2}\right)$ upstream or downstream from the onset point. Wall forcing (e.g. from scaled slot suction or injection $V_{W}$ ) and external forcing (e.g. from a vortical wake outside the boundary layer at $O(1)$ angle $\alpha_{3}$ ) are considered in detail in the Appendix.

are initiated typically close to the onset position, as is the inflectional growth of fixed frequency disturbances in the experiments by Dovgal \& Kozlov (1983, 1995). Hodson's experiments similarly point to substantial 'spot' growth near the onset position. The present theory is local to that position at first before moving on to more global scales downstream. Although viscous Tollmien-Schlichting disturbances may also be present, upstream or downstream, their growth rate is relatively weak and less than the inviscid inflectional growth rate considered herein. So, unless perhaps there is a long region of viscous growth upstream (e.g. as in the fully nonlinear cases considered by Smith \& Bowles 1992; Medeiros \& Gaster 1996; Li et al. 1998; Bowles 2000), such viscous disturbances may be omitted from the present consideration of significant 'spots' arising near a point of minimum basic pressure. The current analysis follows those by Brown \& Smith (1999), by Smith \& Doorly (1992), the latter agreeing experimentally with Clarke et al. (1994) on the 'spot' spread rate versus Mach number, by Bowles \& Smith (1995), which agrees with the observed incompressible spread rate, and by Smith, Dodia \& Bowles (1994) and Smith (1995), which agree on much of the 'spot' flow structure. All those comparisons are for zero pressure gradients and with a parallel-flow approximation. In the adverse-gradient setting, non-parallel effects must be incorporated eventually, and, again, although the linear incompressible range should be examined first, the nonlinear range is also to be included in an attempt at providing insight into the calmed region and other mean-flow alterations.

Section 2 describes the original decelerating boundary layer and the onset of inflectional instability near the pressure minimum. As depicted in figure 1, the scaled streamwise velocity profile $\bar{u}_{0}$ in the boundary layer becomes inflectional there (i.e. at $x=0$ in the figure) and inviscid disturbances generated nearby (within scaled streamwise distances represented by $\sigma^{2}$ ) can grow downstream initially by means of the three-tiered disturbance flow structure shown in the figure. These inviscid disturbances are generated for example by unsteady forcing at the wall such as from 
slot suction and injection, or by unsteady external forcing such as from a relatively thick vortical flow distortion in the stream outside the boundary layer. The initial structure of the inflectional inviscid disturbances leads, in wave terms, to a simple neutral dispersion relation at leading order, over all streamwise and spanwise modes, followed at next order by complex eigenrelations which determine the growth or decay rate over the whole spectrum.

In initial-value terms, concerning a 'spot' disturbance, there is a transient stage first which accommodates the initial conditions as described in $\S 3$, with forced initiation being addressed in the Appendix. Only after the transient stage does a distinct 'spot' really form and grow, as the typical disturbance velocities increase downstream, leading and trailing critical layers emerge from near the wall and adverse pressure gradient effects enter substantially. Their interplay is considered in turn with threedimensionality $(\S 4)$, nonlinearity $(\S \S 5,6)$ and non-parallel effects $(\S 7)$, in the 'spot' evolution.

Section 4 indicates a universal closed planform for the three-dimensional 'spot' at large scaled times, the 'spot' boundary being clear and abrupt. The 'spot' characteristics here are derived from a double steepest-descent approach and are governed by two distinct sets of components, one near-radial from the disturbance initiation point, the other more of a longitudinal-vortex form. Three positions of local maximum fluctuation amplitude occur, one along the 'spot' centreline (towards the leading edge of the 'spot') and the other two off-centre (towards the trailing edge). It may be fortuitous, but, despite the turbulent nature of most 'spot' experiments as opposed to the non-turbulent flow theory, the predicted planform is qualitatively similar, or not dissimilar, to those found in adverse-gradient experiments (Gostelow et al. 1993, 1994; Seifert \& Wygnanski 1995), and likewise for the predicted presence of the off-centre maxima, streamwise vortices and other features (Carlson, Widnall \& Peeters 1982; van Hest 1997).

Section 5 incorporates the first planar nonlinear influence, arising through the moving critical layer which lies effectively at the 'spot' leading edge. This leads to a new vortex roll-up there, within a finite scaled time.

Section 6 considers the corresponding mean-flow alterations, which are found to be different in character ahead of (or underhanging) the 'spot' and behind it. The latter region, possibly modelling the observed calmed region, is controlled mainly by a pressure-feedback mechanism similar to a suggestion by Seifert (private communication 1997), but the former region offers an alternative or supplementary model based on direct inertial forcing and is perhaps closer to an empirical suggestion by Jones (private communication 1997). The mean-flow distortion is found to be amplified by effects acting throughout the region between the leading and trailing critical layers within the 'spot', as the layers depart from the wall downstream. An attached thinned wall layer is left behind the 'spot'. This connects (tentatively) with the observations on calmed region properties by practitioners mentioned earlier in this section.

Section 7 includes direct comments on the results, connections with experiments on 'spots', the extension to non-parallel flow effects, for example, on the approach to flow separation, and brief final points.

\section{The boundary layer, and onset of inflectional instability}

Non-dimensional Cartesian coordinates $x, y$, and $z$ in the streamwise, normal, and spanwise directions, respectively, are to be used, with the corresponding velocity components being $u, v$, and $w$, while $p$ denotes the pressure and $t$ the time. The non- 
dimensionalization is with respect to a characteristic free-stream speed and streamwise length, for example the distance from the leading edge of the blade or airfoil. The Reynolds number $R e$ is large, and the incompressible range is assumed. The resulting basic boundary layer has thickness of order $R e^{-1 / 2}$, say $y=R e^{-1 / 2} \bar{y}$, with stream function $R e^{-1 / 2} \bar{\psi}$, where overbars signify here properties in this undisturbed steady planar boundary layer. The basic boundary-layer flow is considered in $\S 2.1$, particularly near the station of minimum external pressure, and this is followed by discussion in $\S 2.2$ of the onset of inviscidly unstable inflectional modes locally, serving as a guideline for the flow structure in the subsequent examination of freely evolving disturbances.

\subsection{The boundary layer}

The basic flow is a boundary layer, on a solid surface and subject to an $O(1)$ external pressure gradient $\mathrm{d} \bar{p} / \mathrm{d} x$, such that its governing equations are

$$
\bar{u}=\bar{\psi}_{\bar{y}}, \quad \bar{u} \bar{u}_{x}-\bar{\psi}_{x} \bar{u}_{\bar{y}}=-\bar{p}^{\prime}(x)+\bar{u}_{\bar{y} \bar{y}},
$$

the pressure $\bar{p}$ being independent of $\bar{y}$ from the normal momentum balance. Here, $\bar{u}, \bar{\psi}, x, \bar{y}$ are typically of order unity, and

$$
\bar{u}=\bar{\psi}=0 \quad \text { at } \bar{y}=0, \quad \bar{u} \rightarrow \bar{u}_{e}(x) \quad \text { as } \bar{y} \rightarrow \infty,
$$

where $\bar{u}_{e}(x)(>0)$ is the outer stream velocity, satisfying $\bar{u}_{e} \bar{u}_{e}^{\prime}=-\bar{p}^{\prime}$. The prime denotes here ordinary differentiation in $x$ or $\bar{y}$ as appropriate. The scaled skin friction $\bar{\tau}(x) \equiv \partial \bar{u} / \partial \bar{y}(\bar{y}=0)$ is assumed known from the solution of $(2.1 a-d)$ starting at a suitable upstream station, e.g. the leading edge at $x=-\ell_{0}$ where $\ell_{0}$ is $O(1)$ and positive.

At the point of minimum pressure taken to be at $x=0$, the velocity profile is $\bar{u}=\bar{u}_{0}(\bar{y})$ say, with $\bar{u}_{0}^{\prime \prime}(0)=0$ in view of $(2.1 b)$ evaluated at $\bar{y}=0$ and the condition $\bar{p}^{\prime}(0)=0$. We also have $\bar{u}_{0}(0)=0, \bar{u}_{0}^{\prime}(0)=\bar{\lambda}>0$ for attached forward motion, $\bar{u}_{0}^{\prime \prime \prime}(0)=0$ from the $\bar{y}$-derivative of $(2.1 b)$, while $\bar{u}^{\prime v}(0)=-24 \kappa$ with $\kappa$ positive since, from the double $\bar{y}$-derivative of $(2.1 b), \bar{u}^{\prime v}(0)$ equals $\bar{\tau} \mathrm{d} \bar{\tau} / \mathrm{d} x(x=0)$ and, although $\bar{\tau}(0)(=\bar{\lambda})$ is positive, $\mathrm{d} \bar{\tau} / \mathrm{d} x(x=0)$ is expected to be negative because the station $x=0$ lies downstream of a point of maximum favourable pressure gradient. The above near-wall properties tie in with the profile $\bar{u}_{0}$ having negative curvature (no inflection) for all $0<\bar{y}<\infty$, with $\bar{u}_{0}(\infty)=\bar{u}_{e}(0)$ being positive.

Nearby, for small $|x|$, the velocity expansion is regular, of the form

$$
\bar{u}=\bar{u}_{0}(\bar{y})+x \bar{u}_{1}(\bar{y})+\cdots,
$$

in general, and similarly for $\bar{\psi}$, so that from substitution into $(2.1 a-d)$

$$
\bar{u}_{1}=\bar{\psi}_{1}^{\prime}(\bar{y}), \quad \bar{\psi}_{1}=\bar{u}_{0} \int_{0}^{\bar{y}} \bar{u}_{0}^{\prime \prime} \bar{u}_{0}^{-2} \mathrm{~d} \bar{y},
$$

since $\bar{u}_{e}$ is maximal at $x=0$. Hence, locally,

$$
\bar{\tau}(x) \sim \bar{\lambda}-\frac{24 \kappa x}{\bar{\lambda}}, \quad \frac{\partial^{2} \bar{u}}{\partial \bar{y}^{2}}(\bar{y}=0) \sim \bar{p}^{\prime \prime}(0) x,
$$

confirming that $\mathrm{d} \bar{\tau} / \mathrm{d} x$ is negative, whereas the profile curvature at the surface changes sign from negative upstream of $x=0$ to positive downstream, as $\bar{p}^{\prime \prime}(0)>0$ for a pressure minimum. (Also, the boundary-layer displacement can be shown to be increasing with $x$ locally, owing to the negative value of the integral in (2.3) at large $\bar{y}$.) These features enable Rayleigh inflectional inviscid modes to be described, at their onset at $x=0$, as follows. 


\subsection{Onset of instability}

Inviscid instability of the inflectional kind requires solution of the Rayleigh equation, and boundary conditions,

$$
\begin{gathered}
(\bar{u}-c)\left(\tilde{\psi}^{\prime \prime}-\alpha^{2} \tilde{\psi}\right)=\bar{u}^{\prime \prime} \tilde{\psi}, \\
\tilde{\psi}(0)=\tilde{\psi}(\infty)=0,
\end{gathered}
$$

for a sufficiently small planar disturbance $\propto \tilde{\psi}(\bar{y}) \exp [\mathrm{i} \alpha(\hat{x}-c \hat{t})]+C C$ in the stream function, and so on, where $C C$ denotes the complex conjugate, $x=x_{1}+R e^{-1 / 2} \hat{x}$ and time $t \equiv R e^{-1 / 2} \hat{t}$, with $x_{1}$ a constant. (Three-dimensional disturbance properties follow from the planar ones; see later.) This is under the assumption of a parallel basic flow $\bar{u}(\bar{y})$ for now. For the initial-value context of concern later on, the scaled $O(1)$ wavenumber $\alpha$ is real and given, while the scaled $O(1)$ wavespeed $c$ or scaled frequency $\omega \equiv \alpha c$ is unknown and generally complex. Our aim is to find all the wavenumbers for which the flow is unstable, with positive $\omega_{i}$.

Near the pressure-minimum position, $\bar{u}$ is given by (2.1)-(2.4). Use of the parallelflow approximation is justified, however, as we examine disturbances centred at a station $x_{1}$ which is at a small distance of order $\sigma^{2}$ from $x=0$, say $x_{1}=\sigma^{2} \bar{x}_{1}$; the parameter $\sigma$ is small and positive but greater than any inverse power of $R e$ for now. The basic pressure gradient $\mathrm{d} \bar{p} / \mathrm{d} x$ there is small, $\widetilde{g} \sigma^{2}$ say, with $\widetilde{g}\left[\equiv \bar{p}^{\prime \prime}(0) \bar{x}_{1}\right]$ an $O(1)$ constant which has the same sign as $\bar{x}_{1}$, and so $\bar{p}-\bar{p}(0)$ is of order $\sigma^{4}$, whereas $(\bar{\tau}-\bar{\lambda})$ and the surface profile curvature $\partial^{2} \bar{u} / \partial \bar{y}^{2}(\bar{y}=0)$ are both $O\left(\sigma^{2}\right)$ from (2.4). Guided by the Smith \& Bodonyi (1981), Gajjar \& Smith (1985) papers we propose that the unstable range occurs for small wavenumbers with

$$
\alpha=\sigma \alpha_{1}, \quad(c, \omega)=\sum_{n=1}^{4}\left(\sigma^{n} c_{n}, \sigma^{n+1} \omega_{n}\right)+\cdots,
$$

where the coefficients $\alpha_{1}, c_{1}, \omega_{1}$, etc., are of $O(1)$. The solution structure for (2.5) then acquires a three-tiered form (see figure 1) comprising a near-wall sublayer I where $\bar{y}$ is $O(\sigma)$, the main boundary-layer portion II in which $\bar{y} \sim 1$ and an outer tier III where $\bar{y}$ is $O\left(\sigma^{-1}\right)$. The lateral scales for I and III stem, respectively, from the balance of $\bar{u}_{0}^{\prime v}$ and $\bar{u}_{1}^{\prime \prime}$ in the base flow, controlling the height of the inflection point (present for $x_{1}>0$ ), and from the exponential decay in $|\alpha| \bar{y}$ anticipated at large $\bar{y}$.

In tier I, $\bar{y}=\sigma Y$ with $Y \sim 1$ and the basic-flow and perturbation solutions expand as

$$
\begin{aligned}
& \bar{u}=\sigma \bar{\lambda} Y+\sigma^{3} \bar{\lambda}_{1} Y+\sigma^{4} u_{4}(Y)+\cdots, \\
& \tilde{\psi}=\sum_{n=0}^{3} \sigma^{n} \psi_{n}+\cdots
\end{aligned}
$$

Here, $u_{4} \equiv\left(-\kappa Y^{4}+\frac{1}{2} \widetilde{g} Y^{2}\right)$ and $\bar{\lambda}_{1}\left[\equiv-24 \kappa \bar{x}_{1} / \bar{\lambda}\right]$ is an $O(1)$ constant, from (2.2), (2.4). Substitution into $(2.5 a)$ yields the successive solutions

$$
\begin{aligned}
\psi_{n}= & A_{n} \bar{\lambda} Y \quad \text { for } n=0,1,2, \\
\psi_{3}= & A_{3} \bar{\lambda} Y+A_{0}\left[-\kappa\left\{s^{4}+6 \hat{c}_{1} s^{3}+18 \hat{c}_{1}^{2} s^{2}-13 \hat{c}_{1}^{4}\right\}\right. \\
& \left.+\Gamma\left\{s \ln |s|-s+\hat{c}_{1} \ln \hat{c}_{1}-\hat{c}_{1}\right\}+\frac{1}{2} \widetilde{g}\left(s^{2}-\hat{c}_{1}^{2}\right)\right],
\end{aligned}
$$

after $(2.5 b)$ is applied, with $A_{1}-A_{3}$ real without loss of generality, $s \equiv\left(Y-\hat{c}_{1}\right)$, $\Gamma \equiv\left(\widetilde{g}-12 \kappa \hat{c}_{1}^{2}\right) \hat{c}_{1} ; \hat{c}_{1}\left(\equiv c_{1} / \bar{\lambda}\right)$ is found below to be real. The result $(2.8 b)$ holds for 
$s<0$, below the critical level at $Y=\hat{c}_{1}$, whereas above that level the replacement

$$
\ln |s|(\text { for } s<0) \rightarrow \ln s+\mathrm{i} \pi \operatorname{sgn}\left(\alpha_{1}\right) \text { (for } s>0 \text { ), }
$$

is assumed, consistent with a linear critical-layer jump in $\mathrm{d} \tilde{\psi} / \mathrm{d} y$ with $\tilde{\psi}$ continuous. This is verified in subsequent sections. We observe that as $Y \rightarrow \infty$, the dominant imaginary contribution independent of $Y$, in $\tilde{\psi}$, is $-A_{0} \Gamma \hat{c}_{1} \mathrm{i} \pi \sigma^{2} \operatorname{sgn}\left(\alpha_{1}\right)$ from $\psi_{3}$. Hence, in tier II $(\bar{y} \sim 1)$ the perturbation expansion is

$$
\tilde{\psi}=\sum_{n=0}^{4} \sigma^{n-1} \bar{\psi}_{n}+\sigma^{2} \ln \sigma \tilde{\psi}_{3 L}+\cdots,
$$

while $\bar{u}$ is given by (2.2) with $x$ replaced by $\sigma^{2} \bar{x}_{1}$. The two leading terms here are, from $(2.5 a)$ and matching with $(2.8 a)$,

$$
\begin{gathered}
\bar{\psi}_{0}=A_{0} \bar{u}_{0}, \quad \bar{\psi}_{1}=\bar{u}_{0}\left[A_{1}+b_{1} I_{1}\right]-c_{1} A_{0}, \\
I_{1} \equiv-\frac{1}{\bar{\lambda}^{2} \bar{y}}+\int_{0}^{\bar{y}}\left(\frac{1}{\bar{u}_{0}^{2}}-\frac{1}{\bar{\lambda}^{2} \bar{y}^{2}}\right) \mathrm{d} \bar{y},
\end{gathered}
$$

where $b_{1}=-\bar{\lambda} c_{1} A_{0}$. Tier III, in the upper reaches of the boundary layer, has $\bar{y}=\sigma^{-1} \overline{\bar{y}}$ and

$$
\tilde{\psi}=\sigma^{-1} A_{0} \bar{u}_{e}(0) \exp \left(-\left|\alpha_{1}\right| \overline{\bar{y}}\right)+\cdots,
$$

from (2.5a) with $\bar{u}=\bar{u}_{e}(0)$ to leading order and from (2.5c), (2.10a).

Matching of the linearly growing contributions between (2.10b), (2.11) implies that $b_{1}=-A_{0}\left(\bar{u}_{e}(0)\right)^{2}\left|\alpha_{1}\right|$, and hence, with $\gamma_{1} \equiv\left|\alpha_{1}\right|$,

$$
\omega_{1}=\left(\frac{\bar{u}_{e}^{2}(0)}{\bar{\lambda}}\right) \alpha_{1} \gamma_{1}\left[=\alpha_{1} c_{1}\right]
$$

determines the leading-order (real) part of the frequency expansion (2.6). Proceeding to higher order to find the imaginary part, the solution

$$
\bar{\psi}_{4}=\bar{u}_{0}\left[\bar{A}_{4}+b_{4} I_{1}\right]-c_{4} A_{0}+\text { o.t. }
$$

contains the first contribution in tier II that is complex and non-zero as $\bar{y} \rightarrow 0+$, o.t. denoting other real terms. The contribution in $b_{4}$ here grows linearly at large $\bar{y}$ in view of $(2.10 c)$ and so $b_{4}$ must be real in order to match with the exponential forms (as in (2.11)) of the solution in tier III. Hence, matching the imaginary part in (2.13) with that of tier I at large $Y$ yields the value of $c_{4 i}$, from which the dominant growth rate

$$
\omega_{4 i}=\pi \widetilde{g}\left(1-\frac{\gamma_{1}^{2}}{\alpha_{N}^{2}}\right)\left(\frac{\bar{u}_{e}(0)}{\bar{\lambda}}\right)^{4}\left|\alpha_{1}\right| \gamma_{1}^{2}
$$

is obtained, where $\alpha_{N}^{2} \equiv \widetilde{g} \bar{\lambda}^{4} /\left[12 \kappa \bar{u}_{e}^{4}(0)\right]$. The nature of the growth rate (2.14) is consistent with the results in Smith \& Bodonyi (1981), Goldstein, Durbin \& Leib (1987), Gajjar \& Smith (1985).

Regarding the flow structure and results above, we should comment that (i) the disturbance solution depends predominantly only on the quantities $\bar{u}_{e}(0), \bar{\lambda}, \widetilde{g}, \kappa$ of the basic flow, i.e. on the wall and outer-stream properties $\bar{u}_{e}(0), \bar{u}_{e}^{\prime \prime}(0), \bar{\tau}(0), \bar{\tau}^{\prime}(0)$ and on the scaled position $\bar{x}_{1}$, rather than on the entire velocity profile. (ii) A finite range of unstable wavenumbers is induced near the wall, given by $0<\left|\alpha_{1}\right|<\alpha_{N}$ from (2.14), just downstream of the minimum-pressure position. Here, $\alpha_{N}$ is the neutral value. Just upstream, in contrast, $\widetilde{g}$ is negative and $\alpha_{N}^{2}$ must be replaced by $-\alpha_{N}^{2}$, in 
(2.14), so that all wavenumbers are stable there. (iii) The corresponding result for a three-dimensional disturbance has (2.12),(2.14) holding again but $\gamma_{1}^{2} \equiv \alpha_{1}^{2}+\beta_{1}^{2}$, where $\beta=\sigma \beta_{1}$ is the scaled spanwise wavenumber in the extra dependence $\exp (\mathrm{i} \beta \hat{z})$ present then, with $z=R e^{-1 / 2} \hat{z}$. Thus again there is a finite range of unstable wavenumbers, given by $\alpha_{1}^{2}+\beta_{1}^{2}<\alpha_{N}^{2}$ in this case. (iv) The finite range of unstable wavenumbers provoked, in (2.14), and indeed the properties of the whole flow structure above, provide the basis for the study of linear and nonlinear initial-value properties in the following sections. In particular, two timescales are implied almost directly by (2.12), (2.14) with (2.6), one having $\hat{t}$ of order $\sigma^{-2}$ (stage 1 , with $\hat{t}=\sigma^{-2} \tilde{t}$ ), being transient and involving evolution towards the neutral behaviour associated with (2.12), while the other (stage 2, with $\hat{t}=\sigma^{-5} T$ ) has $\hat{t}$ longer, of order $\sigma^{-5}$, leading to slow growth in keeping with (2.14). In the next sections stages 1,2 are examined in turn.

\section{Transient stage}

This first temporal stage is considered now in order to elucidate the emergence of the above Rayleigh behaviour, the transient dependence on the initial conditions and the emerging flow structure for the later 'spot' evolution; see also the Appendix on forced initiation of 'spots' (and the initial-value studies in Stewartson \& Stuart 1971; Chapman \& Proctor 1980; Smith \& Doorly 1992; Jennings, Stewart \& Cowley 1999; Savin et al. 1999; Smith, Bowles \& Li 2000). We note that true disturbance growth or decay, and the formation of a 'spot', come in stage 2 later.

During stage 1 of transients when $\hat{t}=\sigma^{-2} \tilde{t}$ is of order $\sigma^{-2}$ the flow solution is found to remain smooth generally, if the initial conditions are smooth in $\hat{x}\left(=\sigma^{-1} \tilde{x}\right), y$, and no critical layers are induced. Such layers start to emerge at large $\tilde{t}$, however, and have an important bearing on growth and nonlinear effects subsequently, as we will see at the end of this section and thereafter. In the present transient stage the whole solution consisting of the original flow and the perturbations is expanded, within the three tiers I-III and with a perturbation amplitude represented by $\Delta$, and the expansion is substituted into the Navier-Stokes equations. The form involved here is based on interpreting the results in the previous sections in terms of transient times $\tilde{t}$ of $O(1)$. For convenience, the factors $\bar{\lambda}, \bar{u}_{e}(0)$ are taken as unity and $\Delta$ is supposed to be very small, although the influence of increasing its size is first considered at the end of this section. Also, the streamwise coordinate $\tilde{x}$ is of order unity.

\subsection{Disturbance flow structure}

Tier I now has

$$
\begin{aligned}
& u=\sigma Y+\cdots+\sigma^{4} u_{4}(Y)+\cdots+\Delta\left[\sigma^{-1} \tilde{u}_{0}+\cdots+\sigma^{2} \tilde{u}_{3}+\cdots\right], \\
& v=\cdots+\Delta\left[\sigma \tilde{v}_{0}+\cdots+\sigma^{4} \tilde{v}_{3}+\cdots\right], \\
& p=\bar{p}(0)+\frac{1}{2} \bar{p}^{\prime \prime}(0) \bar{x}_{1}^{2} \sigma^{4}+\cdots \Delta\left[\tilde{p}_{0}+\cdots+\sigma^{3} \tilde{p}_{3}+\sigma^{4} \tilde{p}_{4}+\cdots\right],
\end{aligned}
$$

with $\tilde{p}_{0}-\tilde{p}_{3}$ being independent of $Y$. The solutions for the first three orders of approximation of the disturbance give

$$
\tilde{u}_{n}=\tilde{A}_{n}, \quad \tilde{v}_{n}=-\tilde{A}_{n \tilde{x}} Y, \quad \tilde{A}_{n \tilde{t}}=-\tilde{p}_{n \tilde{x}} \quad \text { for } n=0,1,2,
$$

where the $\tilde{A}_{n}$ are real unknown functions of $\tilde{x}, \tilde{t}$. At the next order, the scaled shear $\partial^{2} \tilde{\psi}_{3} / \partial Y^{2}\left(\right.$ where $\tilde{u}_{3}=\partial \tilde{\psi}_{3} / \partial Y$ and $\left.\tilde{v}_{3}=-\partial \tilde{\psi}_{3} / \partial \tilde{x}\right)$ satisfies

$$
\left(\partial_{\tilde{t}}+Y \partial_{\tilde{x}}\right) \tilde{\psi}_{3 Y Y}=u_{4}^{\prime \prime}(Y) \tilde{A}_{0 \tilde{x}} Y,
$$


On 'spot' evolution under an adverse pressure gradient

$$
\begin{gathered}
\tilde{\psi}_{3}=0 \text { at } Y=0, \\
(F . P .) \tilde{\psi}_{3 \tilde{x}}(Y \rightarrow \infty)=\tilde{A}_{3 \tilde{t}},
\end{gathered}
$$

with F.P. denoting here the finite part at large $Y$. The condition $(3.3 b)$ is the usual inviscid one of tangential flow at the solid surface, where there is a thinnner attached Stokes layer to comply with the no-slip surface constraint, while (3.3c) follows from a matching condition with tier II's behaviour.

Tier II is described by

$$
\begin{aligned}
& u=\bar{u}_{0}(\bar{y})+\sigma^{2} \bar{u}_{2}(\bar{y})+\cdots+\Delta\left[\sigma^{-1} \check{u}_{0}+\cdots+\sigma^{2}(\ln \sigma) \check{u}_{3 L}+\sigma^{2} \check{u}_{3}+\cdots\right], \\
& v=\cdots+\Delta\left[\check{v}_{0}+\cdots\right], \quad p=\cdots+\Delta\left[\check{p}_{0}+\cdots\right],
\end{aligned}
$$

where $\check{p}_{0}$ is independent of $\bar{y}$, thus equalling $\tilde{p}_{0}$. The first two orders of solutions now imply that

$$
\begin{aligned}
& \check{u}_{0}=\tilde{A}_{0} \bar{u}_{0}^{\prime}, \quad \check{v}_{0}=-\tilde{A}_{0 \tilde{x}} \bar{u}_{0}, \quad \check{p}_{0}=\tilde{p}_{0}, \\
& \check{u}_{1}=\tilde{A}_{1} \bar{u}_{0}^{\prime}-\left[\bar{u}_{0}^{\prime} I_{1}+\bar{u}_{0}^{-1}\right] \tilde{p}_{0}, \quad \check{v}_{1}=-\tilde{A}_{1 \tilde{x}} \bar{u}_{0}+\left[1+\bar{u}_{0} I_{1}\right] \tilde{p}_{0 \tilde{x}} .
\end{aligned}
$$

The subsequent order of most concern is the one determining $\check{v}_{4}$, which gives the controlling equation $\left(\bar{u}_{0} \partial^{2} / \partial \bar{y}^{2}-\bar{u}_{0}^{\prime \prime}\right) \check{v}_{4}=\cdots+\bar{u}_{0}^{\prime \prime} \partial \tilde{A}_{3} / \partial \tilde{t}$, from which $\check{v}_{4}$ contains a part $\check{v}_{4}=-\partial \tilde{A}_{3} / \partial \tilde{t}$. This verifies the constraint (3.3c) above, which in turn serves to determine $\partial \tilde{A}_{3} / \partial \tilde{t}$ in effect.

Tier III has, since $\bar{u}_{e}(0)$ is unity now,

$$
[u, v, p]=[1,0, \bar{p}(0)]+\cdots+\Delta\left[\hat{u}_{0}, \hat{v}_{0}, \hat{p}_{0}\right]+\cdots,
$$

leading to Laplace's equation in $\tilde{x}, \overline{\bar{y}}$ for each of $\hat{u}_{0}, \hat{v}_{0}$ and $\hat{p}_{0}$. This is subject to far-field decay and to $\hat{p}_{0} \rightarrow \breve{p}_{0}, \partial \hat{p}_{0} / \partial \overline{\bar{y}} \rightarrow \partial^{2} \tilde{A}_{0} / \partial \tilde{x}^{2}$ as $\overline{\bar{y}} \rightarrow 0+$, to merge with the solution of tier II. Therefore, given the pressure balance in (3.5a), the interaction law

$$
\tilde{p}_{0}(\tilde{x}, \tilde{t})=\frac{1}{\pi}(P . V .) \int_{-\infty}^{\infty} \frac{\tilde{A}_{0 \xi} \mathrm{d} \xi}{\tilde{x}-\xi}
$$

is obtained between the surface pressure and the displacement. Here, P.V. denotes the Cauchy principal value.

Coupling (3.7) with (3.2c) for $n=0$ yields a linear integro-differential equation for $\tilde{A}_{0}(\tilde{x}, \tilde{t})$, which governs the leading-order evolution here and which is a linearized Benjamin-Ono equation; see also Zhuk \& Ryzhov (1982) and Smith \& Burrgraf (1985). For input waves of wavenumber $\alpha_{1}$ in $\tilde{x}$, equations (3.2c) and (3.7) also retrieve the formula (2.12) for the main frequency response. For the current initialvalue problem with general smooth bounded input, on the other hand, the solution has

$$
2 \pi \tilde{A}_{0}(\tilde{x}, \tilde{t})=\int_{-\infty}^{\infty} R^{*}(k) \exp [\mathrm{i} k \tilde{x}-\mathrm{i} k|k| \tilde{t}] \mathrm{d} k
$$

with $R^{*} \equiv \tilde{A}_{0}^{*}(k, 0)$ being the initial Fourier transform $(\tilde{x} \rightarrow k$, signified by *). In either case, there is effectively no sustained amplitude growth at this level.

Growth arises in the higher-order terms. Thus, from the Fourier transform solution of $(3.3 a-c)$, we obtain

$$
-\tilde{v}_{3 Y Y}=\left(-12 \kappa Y^{2}+\widetilde{g}\right) \frac{Y}{2 \pi} M, \quad \text { where } M \equiv\left(K_{1}+K_{1}^{C C}\right),
$$

and, with a representative initial disturbance such that $R^{*}=\exp (-q|k|)$, correspond- 
ing to $\tilde{A}_{0}(\tilde{x}, 0) \propto\left(\tilde{x}^{2}+q^{2}\right)^{-1}$, for the sake of argument,

$$
K_{1}(\tilde{x}, Y, \tilde{t}) \equiv \int_{0}^{\infty} \mathrm{i} k \frac{\exp (-q k+\mathrm{i} k \tilde{x})}{(Y-k)}\left\{\exp \left(-\mathrm{i} k^{2} \tilde{t}\right)-\exp (-\mathrm{i} k Y \tilde{t})\right\} \mathrm{d} k
$$

in view of (3.8). Here, $q$ is a prescribed positive constant, and $\tilde{v}_{3 Y Y}$ is assumed to be zero initially. The above confirms that the transient solution is smooth for times $\tilde{t}$ of $O(1)$. The solution is presented in figure 2 , which shows $M$ and $\partial \tilde{v}_{3} / \partial Y$. Figure $2(a)$ gives the temporal development of $M$ at a fixed station $\tilde{x}$ and two values of $Y$. At the same station, figure $2(b)$ shows the profile of $M$ against $Y$, for various times. Figure $2(c)$ presents both $M$ and $\partial \tilde{v}_{3} / \partial Y$ profiles at the downstream moving positions $\tilde{x}=\tilde{t}$. The trend towards a downstream moving 'spot-like' or (to be more specific) confined response at increasing times is notable, and this is explored below. Further, equations (3.9a) and (3.9b) apply whether the disturbance is initiated upstream or downstream of the minimum-pressure point. The latter case, for which $\tilde{g}$ is positive, is shown in the figure. In the case of upstream initiation, the function $M$ (and $K_{1}$ ) stays as shown in the figure, but not $\partial \tilde{v}_{3} / \partial Y$ since $\tilde{g}$ is then negative. The continuous crossover between these two cases is described in $\S 7$. We address the large-time behaviour next.

\subsection{Increasing time}

At large $\tilde{t}$, first (3.8) leads to the downstream response

$$
\pi \tilde{A}_{0} \sim \frac{\pi^{1 / 2} \mathrm{e}^{-q \eta / 2}}{\tilde{t}^{1 / 2}} \cos \left(\frac{\eta^{2} \tilde{t}}{4}-\frac{\pi}{4}\right)
$$

in the representative case, for positive $\tilde{x}$, where $\eta \equiv \tilde{x} / \tilde{t}$ is typically of order unity; upstream, for negative $\tilde{x}$, the response is exponentially small outside a $|\tilde{x}|$ scale of order $\tilde{t}^{1 / 2}$. The lack of a pure wave behaviour here is noteworthy and, to repeat, the solution at this leading order does not depend on whether the relative location $\bar{x}_{1}$ for the initial disturbance is positive (downstream source) or negative (upstream source). The temporal decay in (3.10) contrasts with the later (stage 2) growth within a 'spot' further downstream, as anticipated at the start of this section. It is clear also that two $\tilde{x}$-scales come into play downstream now, one of order $\tilde{t}^{1 / 2}$ because of the cos term above and the other of order $\tilde{t}$ because of the amplitude variation in the exponential terms, these lengthscales agreeing with those of the 'spot'-disturbance analyses in Smith \& Doorly (1992) (see their figure 2), Smith et al. (1994) as well as those in the previous section. Secondly, the function $M$ which is closely related to the vorticity through (3.9a) develops a five-layered structure (i)-(v) within $Y$ of the form, for $\tilde{t}$ large,

$$
\begin{aligned}
& M=o(1) \quad \text { for (i) } 0<Y<\frac{1}{2} \eta \text { and (v) } Y>\eta, \\
& M \sim-4 \pi Y \mathrm{e}^{-q Y} \cos (Y \Phi \hat{t}) \text { for (iii) } \frac{1}{2} \eta<Y<\eta,
\end{aligned}
$$

with $\Phi \equiv(\eta-Y)$ being positive in the main range (3.11b), for $\tilde{x}$ of $O(\tilde{t})$. The solution at this order does depend on the disturbance location $\bar{x}_{1}$ and for now we assume that $\bar{x}_{1}$ is positive. The sketch in figure 3 indicates, in turn, the flow structure at these larger times, the areas of most rapid fluctuation of $M$ in the normal $Y$-direction and in the streamwise $\tilde{x}$-direction, and the evolving structure in the $(\tilde{x}, \tilde{t})$-plane at larger times. Two relatively thin layers (ii) and (iv) join the forms $(3.11 a, b)$ together smoothly, at $Y-\frac{1}{2} \eta=O\left(\tilde{t}^{-1 / 2}\right)$ and $Y-\eta=O\left(\tilde{t}^{-1}\right)$, respectively. Computations at increasing $\tilde{t}$ in figure 2 are in line with this larger-time behaviour, whose details are as follows.

Splitting $K_{1}=L_{a}-L_{b}$ and using P.V. as the limit as $\epsilon \rightarrow 0+$ of the sum of the 

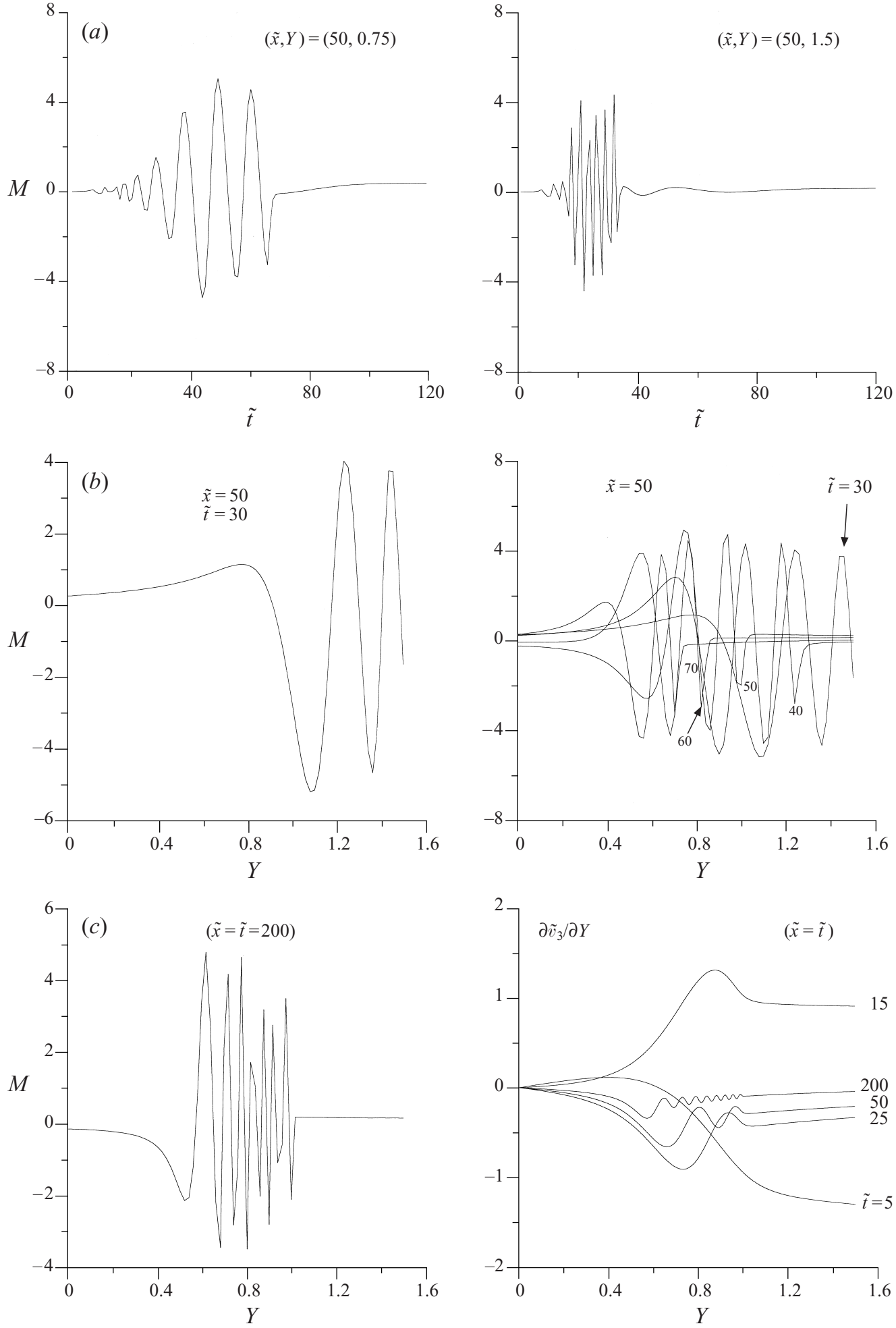

FiguRE 2. Computed behaviour during the transient stage. (a) $M$ vs. $\tilde{t}$ at $\tilde{x}=50$ for $Y=0.75,1.5$. (b) $M$ vs. $Y$ at $\tilde{x}=50$, various $\tilde{t}$. (c) $M$ vs. $Y$ at $\tilde{x}=\tilde{t}=200$, and $\tilde{v}_{3 Y}$ vs. $Y$ along $\tilde{x}=\tilde{t}$ at various $\tilde{t}$. 
180
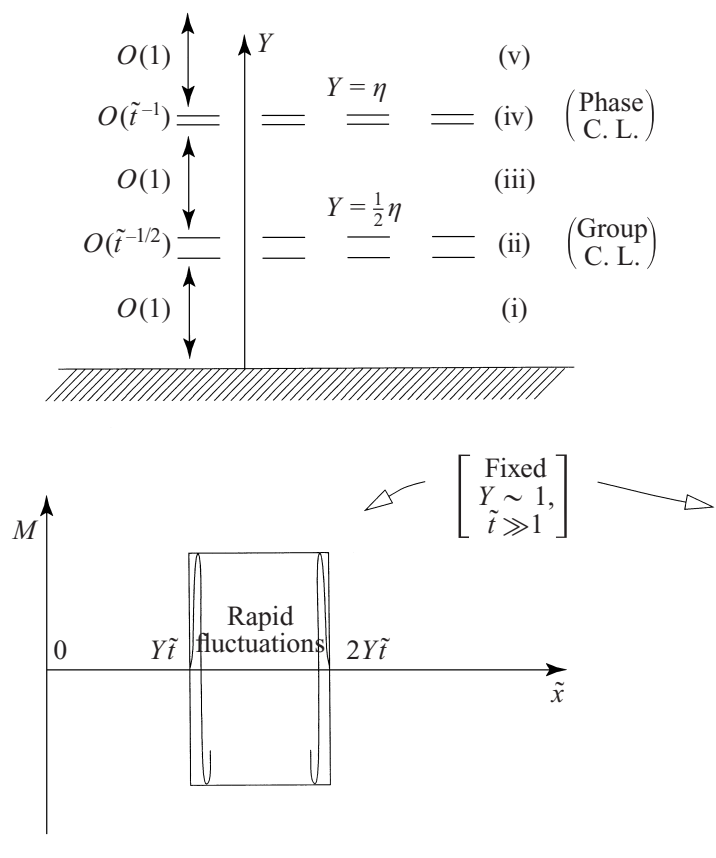

(v)

(iii)
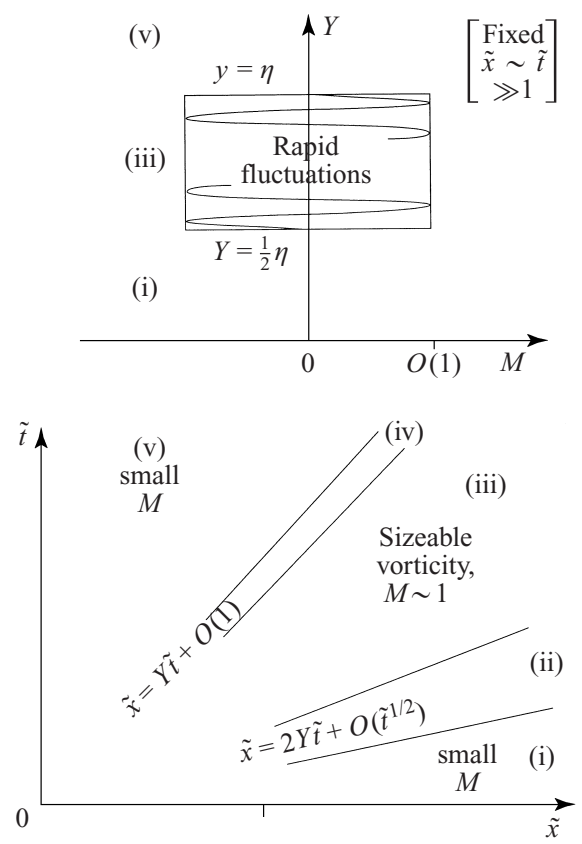

FigURE 3. Structure near the ending of the transient stage, at large $\tilde{t}$, in $\S 3.2$.

integrals from zero to $Y-\epsilon, Y+\epsilon$ to $\infty,(3.9 b)$ becomes

$$
\begin{aligned}
& L_{a}=(P . V .) \int_{0}^{\infty} \frac{\mathrm{i} k \mathrm{e}^{-q k} \mathrm{e}^{\mathrm{i} k \xi+\mathrm{i} k Y \tilde{t}-\mathrm{i} k^{2} \tilde{t}}}{(Y-k)} \mathrm{d} k, \\
& L_{b}=(P . V .) \int_{0}^{\infty} \frac{\mathrm{i} k \mathrm{e}^{-q k} \mathrm{e}^{\mathrm{i} k \xi}}{(Y-k)} \mathrm{d} k,
\end{aligned}
$$

where $\xi \equiv(\tilde{x}-Y \tilde{t})=(\eta-Y) \tilde{t}$. For $\tilde{t} \gg 1$ we examine the layers (i), (iii) and (v) first, where $\xi$ is $O(\tilde{t})$. The major contributions there arise from $k=Y+\bar{k} \tilde{t}^{-1}$ with $\bar{k}$ of $O(1)$, giving $L_{a} \sim \pi Y \exp \{-q Y+\mathrm{i}(\eta-Y) Y \tilde{t}\}$ multiplied by $\operatorname{sgn}(\eta-2 Y)$, and similarly for $L_{b}$. Hence,

$$
M \sim 2 \pi Y \mathrm{e}^{-q Y} \cos [Y(\eta-Y) \tilde{t}]\{\operatorname{sgn}(\eta-2 Y)-\operatorname{sgn}(\eta-Y)\},
$$

with relative error of order $\tilde{t}^{-1}$. This leads to the results $(3.11 a, b)$ to lowest order. More precisely in layers (i) and (v), however, the main non-zero parts are from stationary-phase properties in $L_{a}$, concentrated at the values $k=\frac{1}{2} \eta+O\left(\tilde{t}^{-1 / 2}\right)$, which yield

$$
M \sim\left(\frac{\pi}{\tilde{t}}\right)^{1 / 2} \frac{\eta \mathrm{e}^{-q \eta / 2}}{(Y-\eta / 2)} \cos \left(\frac{\eta^{2} \tilde{t}}{4}+\frac{\pi}{4}\right) .
$$

The $(Y-\eta / 2)^{-1}$ singularity which clearly occurs at the top of layer (i) here is consistent with an $O\left(Q^{-1}\right)$ term present in (ii), at large $Q$ in (3.15a) below, and it demonstrates the emergence of a critical layer at large times, with $M$ becoming $O(1)$ where $Y-\eta / 2$ is $O\left(\tilde{t}^{-1 / 2}\right)$. Layer (ii) is defined by $Y=\frac{1}{2} \eta+\tilde{t}^{-1 / 2} Q$ say with $Q$ of $O(1)$. Here, the major parts of the integrals in $(3.12 a, b)$ stem from $k=\frac{1}{2} \eta+\tilde{t}^{-1 / 2} \bar{s}$ 
On 'spot' evolution under an adverse pressure gradient

and $k=\frac{1}{2} \eta+\tilde{t}^{-1 / 2} Q+\tilde{t}^{-1} r$ in turn, with $\bar{s}, r \sim 1$, so that

$$
\begin{aligned}
L_{a} & \sim \frac{1}{2} \mathrm{i} \eta \exp \left[-\frac{1}{2} q \eta+\frac{1}{4} \mathrm{i} \eta^{2} \tilde{t}\right](P . V .) \int_{-\infty}^{\infty} \frac{\exp \left(-\mathrm{i} \bar{s}^{2} \mathrm{~d} \bar{s}\right)}{(Q-\bar{s})}, \\
L_{b} & \sim \frac{1}{2} \pi \eta \exp \left[-\frac{1}{2} q \eta+\mathrm{i}\left(\frac{1}{4} \eta^{2} \tilde{t}-Q^{2}\right)\right]
\end{aligned}
$$

are odd and even, respectively, in $Q$. Therefore,

$$
M \sim-\pi \eta \mathrm{e}^{-q \eta / 2} \cos \left(\frac{1}{4} \eta^{2} \tilde{t}-Q^{2}\right)+F(Q),
$$

with the function $F(Q)$ being odd in $Q$, from which integration in $Q$ gives

$$
\int_{-\infty}^{\infty} M \mathrm{~d} Q \sim-\pi^{3 / 2} \eta \mathrm{e}^{-q \eta / 2} \cos \left(\frac{\eta^{2} \tilde{t}}{4}-\frac{\pi}{4}\right)[\equiv \tilde{b}] .
$$

In layer (iv), $Y=\eta+\tilde{t}^{-1} \widetilde{\xi}$ and $\widetilde{\xi} \sim 1$, leaving $\xi=-\widetilde{\xi}$ of $O(1)$. The dominant parts of $(3.12 a, b)$ come from $k=\eta+\tilde{t}^{-1}(\widetilde{\xi}+\bar{k})$ and $k \sim 1$, respectively, yielding

$$
L_{a} \sim-\pi \eta \mathrm{e}^{-q \eta-\mathrm{i} \eta \tilde{\xi}}, \quad L_{b} \sim(P . V .) \int_{0}^{\infty} \frac{\mathrm{i} k \mathrm{e}^{-q k-\mathrm{i} k \tilde{\xi}}}{(\eta-k)} \mathrm{d} k .
$$

So

$$
M \sim-2 \pi \eta \mathrm{e}^{-q \eta} \cos (\eta \widetilde{\xi})-2(P . V .) \int_{0}^{\infty} \frac{k \mathrm{e}^{-q k}}{(\eta-k)} \sin (k \widetilde{\xi}) \mathrm{d} k,
$$

the first contribution being even in $\widetilde{\xi}$ and the second odd, and in consequence

$$
M \rightarrow 0 \quad \text { as } \widetilde{\xi} \rightarrow+\infty, \quad M \sim-4 \pi \eta \mathrm{e}^{-q \eta} \cos (\eta \widetilde{\xi}) \quad \text { as } \widetilde{\xi} \rightarrow-\infty .
$$

These asymptotes merge with the solutions in layers (v) and (iii) in turn, while the integral of $M$ with respect to $Y$ across (iv) is approximately $O\left(\tilde{t}^{-1}\right)$. See figure 3 again.

It follows that two critical layers (ii) and (iv) are emerging at large times $\tilde{t}$, associated with the phase velocity and the group velocity, respectively, each layer at first lying close to the wall at distances $\tilde{x}$ of $O\left(\tilde{t}^{1 / 2}\right)$, but leaving the wall on the $\tilde{x} \sim \tilde{t}$ scale downstream. The former layer (which is the leading layer, see figure 3) turns out to be the vital one. Integrating $Y u_{4}^{\prime \prime} M$ in $Y$ to derive $\partial \tilde{v}_{3} / \partial Y$ from (3.9a), we see that $(3.15 d)$ is indeed dominant, producing the form

$$
\tilde{v}_{3 Y}-\tilde{v}_{3 Y}(Y \rightarrow \infty)=\tilde{a} \text { for } 0<Y<\frac{1}{2} \eta, \quad \text { zero for } Y>\frac{1}{2} \eta,
$$

where

$$
\tilde{a} \equiv \frac{\tilde{b} \tilde{t}^{-1 / 2}}{2 \pi}\left[Y u_{4}^{\prime \prime}(Y)\right]_{Y=\frac{1}{2} \eta}
$$

to order $\tilde{t}^{-1 / 2}$. Here, we may regard $\tilde{v}_{3 Y}(Y \rightarrow \infty)$ as zero for the significant part of the disturbance, although, in fact, an unknown constant can be added to $\tilde{v}_{3 Y}$ which along with terms similar to those in $(2.8 b)$ does not affect the subsequent analysis. The main contribution to $\tilde{v}_{3 Y}$ in $(3.17 a)$ is clearly due to the jump across layer (ii), the jumps across other layers being $o\left(\tilde{t}^{-1 / 2}\right)$. Hence (3.17a) implies the leading-order result

$$
\tilde{v}_{3}=\tilde{a} Y \quad \text { for } 0<Y<\frac{1}{2} \eta, \quad \widetilde{v_{3}}=\frac{1}{2} \tilde{a} \eta \quad \text { for } Y>\frac{1}{2} \eta,
$$

since $\tilde{v}_{3}$ must be zero at $Y=0$. It follows also that, for large $\tilde{t}$, the dominant growth 
is given by

$$
\tilde{A}_{3 \tilde{t}}=-\frac{1}{2} \tilde{a} \eta=\left(\frac{\pi}{\tilde{t}}\right)^{1 / 2} \frac{\eta^{3} \mathrm{e}^{-q \eta / 2} u_{4}^{\prime \prime}\left(\frac{1}{2} \eta\right)}{8} \cos \left(\frac{\eta^{2} \tilde{t}}{4}-\frac{\pi}{4}\right)
$$

from (3.3c). The growth rate $\left(\partial \tilde{A}_{3} / \partial \tilde{t}\right) / \tilde{A}_{0}$ inferred from (3.19) with (3.10) is exactly as in (2.14) but evaluated at $\alpha_{1}=\frac{1}{2} \eta$, in line with the stationary-phase analysis of $\tilde{A}_{0}$ leading to (3.10). All the results also generalize, with $\exp \left(-\frac{1}{2} q \eta\right)$ above then being replaced by the amplitude of $R^{*}\left(\frac{1}{2} \eta\right)$ throughout, accompanied by a phase shift from the argument of $R^{*}$. We remark further that the dependence on $Y u_{4}^{\prime \prime}$ in (3.9a) and (3.17b) and implicit in (3.19) agrees with that in Smith et al. (2000). The prime point nevertheless is that, as might be expected, the phase-velocity values of the profile curvature $u_{4}^{\prime \prime}\left(\frac{1}{2} \eta\right)$ and the corresponding moving critical layer (ii) become the controlling feature at large $\tilde{t}$, just prior to 'spot' formation. Thus, the relevance of the approach in $\S 2$ is confirmed. Similar working holds for three-dimensional disturbances, leading to $\S 4$ below. Indeed, in the subsequent temporal stage studied in $\S 4$ ff the 'spot' becomes truly distinct and its centre where maximum amplitude growth occurs can be identified clearly, downstream of the minimum pressure position.

In anticipation of $\S 5$, an estimate may now be made for the first occurrence of significant nonlinear effects as the input disturbance amplitude $\Delta$ is increased, based on the above behaviour during stage 1 . In view of (3.17a)-(3.19), we expect such nonlinearity to enter first within layer (ii) during the subsequent stage 2 which has already been identified with the long timescale $\tilde{t} \sim \sigma^{-3}$. The representative inertial operator in (ii) is of order $\left(\sigma \tilde{t}^{-1 / 2}\right)$ times $\left(\sigma R e^{1 / 2}\right)$ in the linear regime, from $(u-c) \partial / \partial x$ with $(u-c) \sim \sigma\left(Y-Y_{g}\right)$ from (3.1a) and (3.17a), where $Y_{g} \equiv \frac{1}{2} \eta$, and $\partial / \partial x \sim \sigma R e^{1 / 2}$ owing to the oscillatory response in (3.10) and (3.19), along with the $O\left(\tilde{t}^{-1 / 2}\right)$ thickness of layer (ii). This is to be compared with the operator $v \partial / \partial y$, to obtain the new nonlinear regime, indicating the typical order $\left(\Delta \sigma \tilde{t}^{-1 / 2}\right)$ times $\left(R e^{1 / 2} \sigma^{-1} \tilde{t}^{1 / 2}\right)$, since $v$ is given by $(3.1 b),(3.2)$ with (3.10). The two operators therefore become comparable when $\sigma^{2} \tilde{t}^{-1 / 2} R e^{1 / 2} \sim \Delta R e^{1 / 2}$, i.e. when $\Delta \sim \sigma^{2} \tilde{t}^{-1 / 2}$. Allowing for the relative timescale $\sigma^{-3}$ of stage 2 , then, the suggestion for the lowest disturbance size to provoke substantial nonlinearity is

$$
\Delta=O\left(\sigma^{7 / 2}\right),
$$

for stage 2. This estimate is used in $\S 5$. For lower amplitudes,

$$
\Delta \ll \sigma^{7 / 2} \text {, }
$$

and linear theory continues to hold.

\section{Three-dimensional 'spot'}

For amplitude levels satisfying (3.21) the evolution of the three-dimensional disturbance during stage 2 (subsequent to the transient stage 1 of the previous section) is given by a double Fourier $\left({ }^{* *}\right)$ inversion,

$$
4 \pi^{2} \widetilde{Q}(\widetilde{x}, \widetilde{z}, \widetilde{t})=\iint \widetilde{Q}^{* *}(k, \ell, 0) \exp (K) \mathrm{d} k \mathrm{~d} \ell,
$$

with, from (2.12) and (2.14),

$$
\begin{aligned}
K(k, \ell, \widetilde{x}, \widetilde{z}, \widetilde{t}) \equiv & {\left[\mathrm{i} k \hat{X}+\mathrm{i} \ell \hat{Z}-\mathrm{i} k\left(k^{2}+\ell^{2}\right)^{1 / 2}\right.} \\
& \left.+\hat{\mathrm{g}}\left\{1-\left(k^{2}+\ell^{2}\right) / \alpha_{N}^{2}\right\}|k|\left(k^{2}+\ell^{2}\right)\right] \widetilde{t} .
\end{aligned}
$$


Here, time $\widetilde{t} \gg 1$ but $\hat{g} \widetilde{t} \sim 1$, where $\hat{g} \equiv \sigma^{3} \pi \widetilde{g}$ is small and positive here, while $(\hat{X}, \hat{Z})=(\widetilde{x}, \widetilde{z}) / \widetilde{t}$ are $O(1)$ and $k, \ell$ are written for $\alpha_{1}, \beta_{1}$, respectively. The integral in $(4.1 a)$, with real $k, \ell$, is taken over positive $k$ because of symmetry. The initial condition $\widetilde{Q}^{* *}(k, \ell, 0)$ is $\exp \left(-q\left(k^{2}+\ell^{2}\right)^{1 / 2}\right)$ for example.

To find the 'spot' behaviour at large times $\widehat{g} \widetilde{t}$ we seek extrema of $K$ with respect to $k, \ell$ based on a double steepest-descent method (Brown \& Smith 1999). Thus, zeros of $\partial K / \partial \ell$ require

$$
\mathrm{i} \hat{Z}=\mathrm{i} k \ell\left(k^{2}+\ell^{2}\right)^{-1 / 2}-2 \hat{\mathrm{g}} \ell|k|\left\{1-2\left(k^{2}+\ell^{2}\right) \alpha_{N}^{-2}\right\},
$$

determining $\ell(k)$. The value of $K$ then is as in $(4.1 b)$ with $\ell=\ell(k)$. Hence, zeros of $\partial K / \partial k$ require also that

$$
\begin{aligned}
\mathrm{i} \hat{X}+\mathrm{i} \ell^{\prime} & \hat{Z}-\mathrm{i}\left(k^{2}+\ell^{2}\right)^{1 / 2}-\mathrm{i} k\left(k+\ell \ell^{\prime}\right)\left(k^{2}+\ell^{2}\right)^{-1 / 2} \\
& +\hat{g}\left[\left(k^{2}+\ell^{2}\right)-\left(k^{2}+\ell^{2}\right)^{2} \alpha_{N}^{-2}+2 k\left(k+\ell \ell^{\prime}\right)\left\{1-2\left(k^{2}+\ell^{2}\right) \alpha_{N}^{-2}\right\}\right]=0,
\end{aligned}
$$

where $\ell^{\prime}$ denotes $\mathrm{d} \ell / \mathrm{d} k$. So, in principle, $(4.2 b)$ determines $k$ extremals, with the exponentially large contribution $\operatorname{Re}(K / \widetilde{t})$ then to be evaluated using $(4.1 b)$. Here, we recall that $\operatorname{Re}\left(k^{2}+\ell^{2}\right)^{1 / 2}$ must be positive. In addition, however, $\hat{g}$ is small, so that $(k, \ell)=\left(k_{0}, \ell_{0}\right)+\hat{g}\left(k_{1}, \ell_{1}\right)+\cdots$ can be substituted into the above. This leaves the relations

$$
\begin{gathered}
\ell_{0}=\frac{k_{0} \hat{Z}}{\left(k_{0}^{2}-\hat{Z}^{2}\right)^{1 / 2}}, \quad 8 k_{0}^{2}=\left(4 \hat{Z}^{2}+\hat{X}^{2}\right) \pm \hat{X}\left(\hat{X}^{2}-8 \hat{Z}^{2}\right)^{1 / 2}, \\
\ell_{0}^{\prime}\left[\equiv \mathrm{d} \ell_{0} / \mathrm{d} k_{0}\right]=-\hat{Z}^{3}\left(k_{0}^{2}-\hat{Z}^{2}\right)^{-3 / 2},
\end{gathered}
$$

for the extremal values $k_{0}, \ell_{0}$ (Smith et al. 1994), and the equations

$$
S_{1} \bar{k}_{1}+S_{2} \bar{\ell}_{1}=S_{3}, \quad S_{4} \bar{k}_{1}+S_{5} \bar{\ell}_{1}=S_{6}
$$

for $\bar{k}_{1}, \bar{\ell}_{1}$, where the extremals are $k_{1}=\mathrm{i} \bar{k}_{1}, \ell_{1}=\mathrm{i} \bar{\ell}_{1}$ and, with $B_{0} \equiv\left(k_{0}^{2}+\ell_{0}^{2}\right)$,

$$
\begin{aligned}
& S_{1}=\hat{Z} k_{0} B_{0}^{-1 / 2}-b_{0}, \quad S_{2}=\hat{Z} \ell_{0} B_{0}^{-1 / 2}-k_{0}, \\
& S_{3}=2 k_{0} \ell_{0} B_{0}^{1 / 2}\left(1-2 B_{0} \alpha_{N}^{-2}\right), \\
& S_{4}=-\left(3 k_{0}+\ell_{0} \ell_{0}^{\prime}\right) B_{0}^{-1 / 2}+k_{0}^{2}\left(k_{0}+\ell_{0} \ell_{0}^{\prime}\right) B_{0}^{-3 / 2}, \\
& S_{5}=-\left(\ell_{0}+k_{0} \ell_{0}^{\prime}\right) B_{0}^{-1 / 2}+k_{0} \ell_{0}\left(k_{0}+\ell_{0} \ell_{0}^{\prime}\right) B_{0}^{-3 / 2}, \\
& S_{6}=B_{0}\left(1-B_{0} \alpha_{N}^{-2}\right)+2 k_{0}\left(k_{0}+\ell_{0} \ell_{0}^{\prime}\right)\left(1-2 B_{0} \alpha_{N}^{-2}\right) .
\end{aligned}
$$

The ensuing exponent (growth) term is given by

$$
\begin{aligned}
E\left[\equiv \frac{\operatorname{Re}(K)}{\hat{g} \widetilde{t}}\right]=-\bar{k}_{1}\left\{\hat{X}-\left(2 k_{0}^{2}+\ell_{0}^{2}\right) B_{0}^{-1 / 2}\right\}-\bar{\ell}_{1}\{\hat{Z}- & \left.k_{0} \ell_{0} B_{0}^{-1 / 2}\right\} \\
& +\left(1-B_{0} \alpha_{N}^{-2}\right) k_{0} B_{0}
\end{aligned}
$$

since $K$ is purely imaginary to leading order. The disturbance $\widetilde{Q}$ is therefore proportional to $\widetilde{Q}^{* *}\left(k_{0}, \ell_{0}, 0\right) \exp (E \hat{\mathrm{g}} \widetilde{t}) / \widetilde{t}$ whenever $E$ is positive, at large $\hat{\mathrm{g}} \widetilde{t}$ times.

Computations were performed as follows. For specified $\hat{X}, \hat{Z}$ values, and with $\alpha_{N}^{2}$ set as 2 without loss of generality, $k_{0}, \ell_{0}, \ell_{0}^{\prime}$ are evaluated successively from $(4.3 b, a, c)$, then the corresponding $S_{1}-S_{6}$ from $(4.5 a-f), \bar{k}_{1}, \bar{\ell}_{1}$ from solving $(4.4 a, b)$, and, hence, 

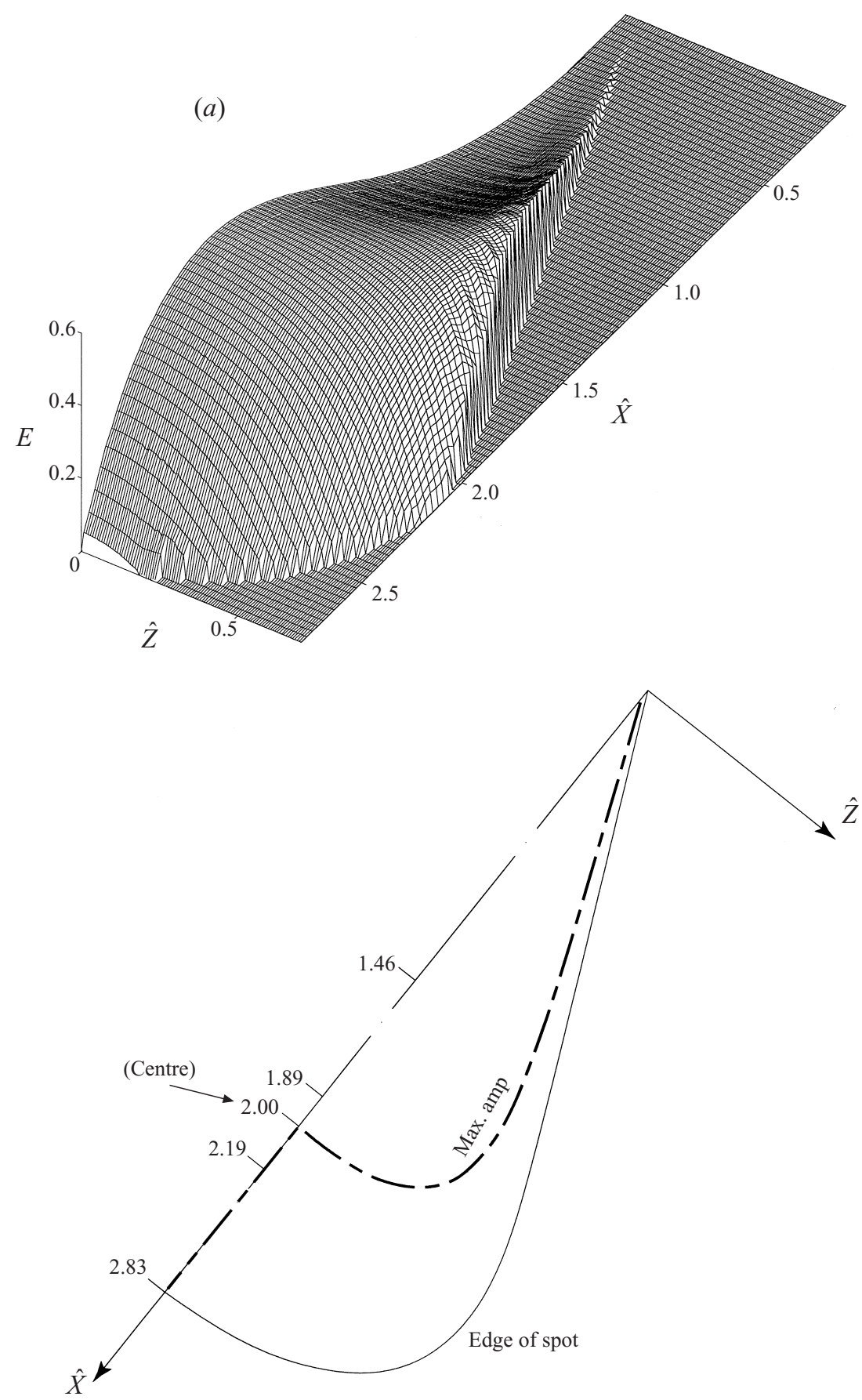

FigURE 4. For the three-dimensional 'spot': (a), sketch of 'spot' planform (lower diagram), with significant $\hat{X}$ values and properties, and surface plot (upper diagram) of $E$ against $\hat{X}, \hat{Z} ;(b)-(g)$, computed growth $E$ vs. $\hat{Z}$ at stations $\hat{X}=2.8,2.5,2,1.5,1,0.8$, respectively, showing both $(+)$ and $(-)$ components; $(h)$, limit solution near 'spot' trailing edge. See $\S 4$. 
(b)

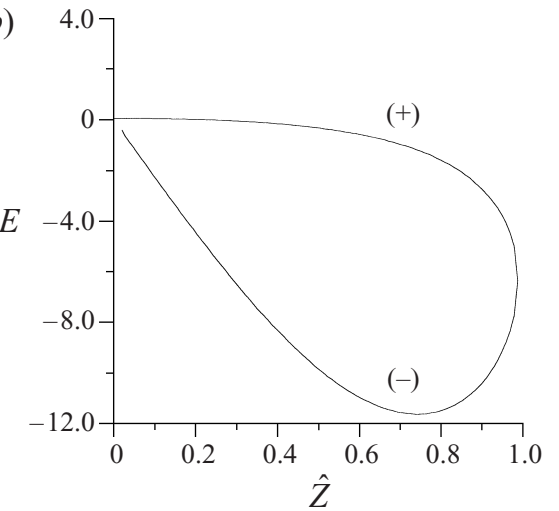

(d)

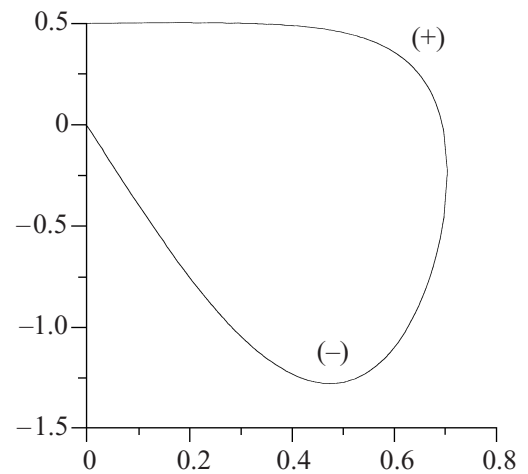

(f)

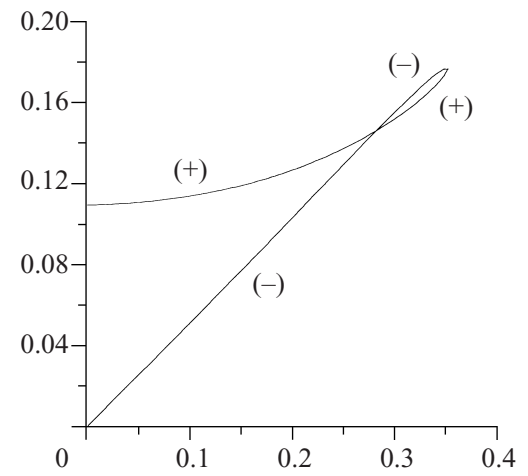

(c) $2.0-$

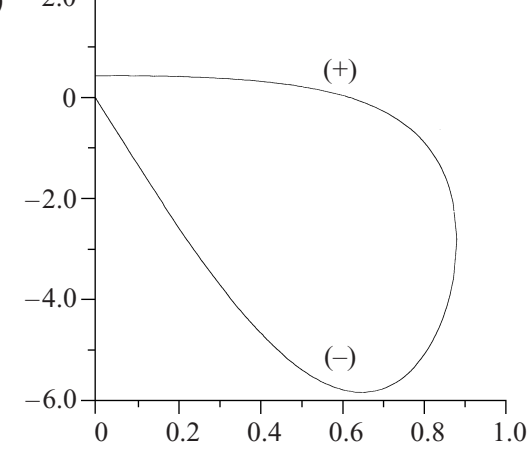

(e)

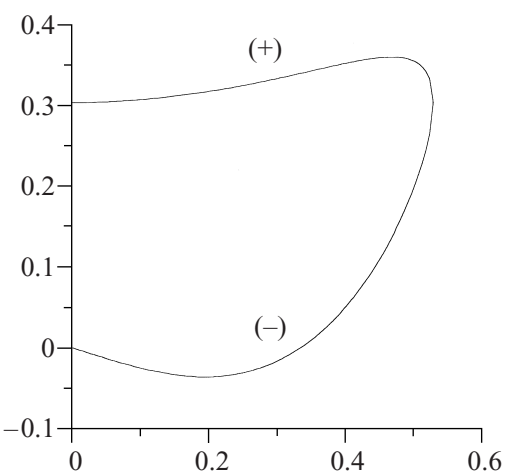

$(g)$

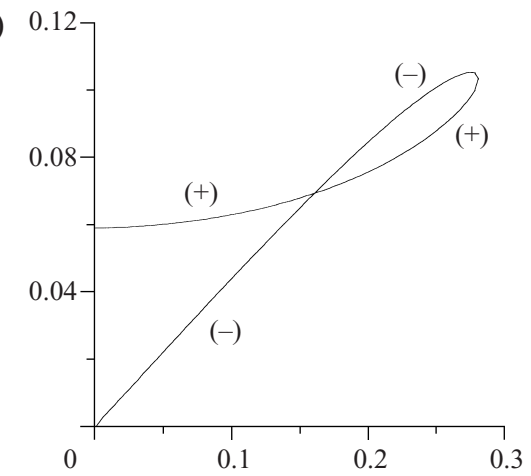

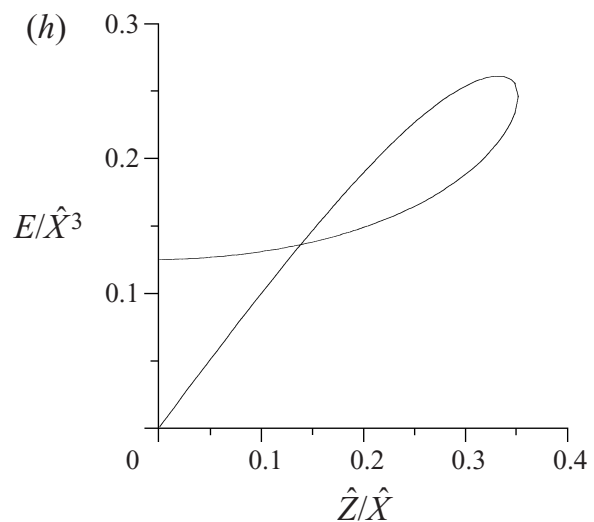

FigURE $4(b-h)$. For caption see facing page. 
$E$ is determined from (4.6). This is repeated for all $(\hat{X}, \hat{Z})$ points of concern, to enable plotting of $E(\hat{X}, \hat{Z})$. Symmetry confines the task to $\hat{Z} \geqslant 0$. Also $\hat{X} \geqslant 0$ only. Further, the computations are done for each sign in $(4.3 b)$ (yielding $( \pm)$ contributions in turn), to examine which yields the larger contribution to $E$.

Numerical results, an exponent surface plot and a summary sketch are presented in figure 4 . The 'spot' within which $E$ is positive has a closed planform in $\hat{X}, \hat{Z}$, with its spanwise edge (side) straight along $\hat{Z}=8^{-1 / 2} \hat{X}$ between the spot trailing edge $\hat{X}=0+$ and the station $\hat{X}=1.8856 \ldots(=4 \sqrt{2} / 3)$ before curving smoothly to form a rounded leading edge at $\hat{X}=2.828 \ldots(=2 \sqrt{2}), Z=0$, with symmetry about the $\hat{X}$-axis. Along the straight portion of the spanwise edge, which produces a spread half-angle of $19.47^{\circ}$, the $( \pm)$ contributions to $E$ are equal and positive, reaching a maximum at $\hat{X}=1.4606 \ldots$, whereas along the curved portion $E$ is zero. Outside, $E$ is negative. The $(+)$ contribution dominates along the 'spot' centreline $\hat{Z}=0$, inducing an overall maximum value of $E$ at $\hat{X}=2.191 \ldots(=2 \sqrt{6 / 5})$. On the other hand, plotting at each $\hat{X}$ station the position $\hat{Z}$ which gives the maximum $E$ value, we obtain a curve (within the 'spot') which originates from the trailing edge at an inclination between $0^{\circ}$ and $19.47^{\circ}$ but bends smoothly and gradually towards the $\hat{X}$-axis as $\hat{X}$ increases. The curve then intersects the $\hat{X}$-axis normally, at $\hat{X}=2$, before remaining along that axis for $2 \leqslant \hat{X} \leqslant 2.828 \ldots$. Thus, moving backwards from the leading edge, we see first in figure $4(b)(\hat{X}=2.8)$ that the maximum $E$ value is at the centreline, while the 'spot' edge spanwise is at $\hat{Z} \approx 0.20$ where $E$ passes regularly through zero. In figure $4(c)$, where $\hat{X}=2.5$, the edge in $\hat{Z}$ increases to approximately 0.6. Figure $4(d)(\hat{X}=2)$ shows the maximum still just at the centreline, the $\hat{Z}$ edge value being about 0.7 . Then, figure $4(e)(\hat{X}=1.5)$ indicates that the maximum position is moved off-centre, remaining distinct from the (reduced) edge position $\hat{Z} \approx 0.5$, at which $E$ is now non-zero, and that the $(-)$ component is now positive, but still less than the $(+)$ component. In figure $4(f)(\hat{X}=1)$, however, the off-centre maximum is due to the $(-)$ contribution, overwhelming the $(+)$ contribution at $\hat{Z} \approx 0.28$, quite near the spanwise edge position $\hat{Z} \approx 0.34$. Figure $4(g)$, for $\hat{X}=0.8$, shows a similar but more pronounced crossover, the spanwise edge position having now decreased to about 0.28 . Figure $4(h)$ presents the limit solution as $\hat{X} \rightarrow 0, \hat{Z} \rightarrow 0$ (trailing edge, see below), which confirms the nearby trends.

Analytical properties from (4.3a)-(4.6) are useful for comparisons. (i) $|\hat{Z}|<\hat{X} / \sqrt{8}$ to keep $k_{0}$ real in $(4.3 b)$. (ii) Along the straight portion $\hat{Z}=\hat{X} / \sqrt{8}(-)$ of the spanwise edge, the form of $E$ is $9 \hat{X}^{3} \sqrt{3}\left(1-9 \hat{X}^{2} / 32\right) / 64$, this expression yielding the maximum spanwise edge value $0.30358 \ldots$ at $\hat{X}=1.4606 \ldots(=4 \sqrt{2 / 15})$ and the cut-off at $\hat{X}=1.8856 \ldots$ mentioned above. The merging between these positive $E$ values along the straight portion and the zero/negative values outside the 'spot' occurs in a thinner layer, as in Smith \& Doorly (1992). (iii) Analysis of the (+) contribution for $\hat{Z} \rightarrow 0$ shows that along the $\hat{X}$-axis $E$ is given by

$$
\left(\frac{1}{2} \hat{X}\right)^{3}\left(1-\hat{X}^{2}\left(4 \alpha_{N}^{2}\right)^{-1}\right) \text {. }
$$

This is in line with the absolute maximum $0.52581 \ldots(=12 \sqrt{6} / 5 \sqrt{5})$ of $E$ occurring along the $\hat{X}$-axis at $\hat{X}=2.191 \ldots$ (which can be regarded as the 'centre' of the 'spot') and with the 'spot' trailing- and leading-edge positions earlier. (iv) Near the leading edge $E \sim 4 \sqrt{2}-2 \hat{X}-\sqrt{2} \hat{Z}^{2}$, from the $(+)$ contribution, and so the 'spot' spanwise edge locally has the parabolic shape $\hat{Z} \sim 2^{1 / 4}(2 \sqrt{2}-\hat{X})^{1 / 2}$. (v) Near $\hat{Z}=0$, the modification to $\hat{E}$ in (4.7) is $\frac{1}{2} \hat{X}\left(1-\frac{1}{4} \hat{X}^{2}\right) \hat{Z}^{2}+\cdots$, confirming that the spanwise maximum $E$ value is along the centreline for $\hat{X}>2$ but off-centre if $\hat{X}<2$. 
(vi) Near the trailing edge as $(\hat{X}, \hat{Z}) \rightarrow(0,0)$, the quantities $k_{0}, \ell_{0}, \ell_{0}^{\prime}, S_{1}-S_{6}, \bar{k}_{1}, \bar{\ell}_{1}$ scale as $\hat{X}, \hat{X}, 1, \hat{X}, \hat{X}, \hat{X}^{3}, 1,1, \hat{X}^{2}, \hat{X}^{2}, \hat{X}^{2}$, respectively, so that $E \propto \hat{X}^{3}$ for both $( \pm)$ contributions. The scaled quantities satisfy $(4.3 a)-(4.6)$ in full, except that $\alpha_{N}^{-2}$ is replaced by zero, in effect. Graphs of the scaled quantities are in figure $4(h)$. (vii) The $(-)$ contribution for small $\hat{Z}$ is found to produce the response $\hat{X}^{2}\left(1-\frac{1}{2} \hat{X}^{2}\right) \hat{Z}$ for $E$ to leading order (thus implying a thinner symmetry-achieving region in which $\widetilde{z}$ is $O\left(\hat{\mathrm{g}}^{-1}\right)$ at large $\hat{\mathrm{g}} \widetilde{t}$ for the $(-)$ contribution, although the $(+)$ contribution then dominates locally anyway). All the above analytical properties agree well with the computational results in figure 4.

A crossover is evident at comparatively small $\hat{X}$ stations in figure 4 , from the $(+)$ contribution being the greater to the $(-)$ one dominating, as $\hat{Z}$ increases. To verify the crossover and the resulting discontinuity in the slope of $E$ versus $\hat{Z}$, we performed direct computations of the integral $(4.1 a)$ at several $\hat{Z}$ values. In particular, for the value $(\hat{X}, \hat{Z})=(0.8,0.25)$ (see figure $4 g$ ) the integral was first evaluated over a large $(k, \ell)$ domain, then over a smaller domain containing the two $\left(k_{0}, \ell_{0}\right)$ positions implied by the $(+)$ and $(-)$ signs, and, finally, over two still smaller domains each containing only one of the $( \pm)$ positions $\left(k_{0}, \ell_{0}\right)$. This was for time $\widetilde{t}=500$ as a typical case, with $\hat{g}$ taken as 0.1 . The four computations agreed closely except for those concentrated relatively near the $(+)$ position, thus pointing to the predicted dominance of the $(-)$ contribution; and the results were in close agreement with the growth factor inferred from figure $4(\mathrm{~g})$.

The $(+)$ contribution is clearly the overriding one in most areas of the 'spot', including the curved portion of the 'spot' side and the centreline. The $(-)$ contribution is nevertheless of interest as it controls the area near the 'spot' sides upstream of the 'spot' centre and it is more of longitudinal-vortex form at heart than radial-wave form, at least in the limit of small $\hat{Z}$ (see analytical properties above), and so is distinct from the $(+)$ contribution. This should be compared with Brown \& Smith's (1999) case, where the (+) type is everywhere dominant in the 'spot'. Also, to a certain extent, the planform resembles that of the channel-flow 'spots' of Carlson et al. without adverse pressure gradients, although this must be regarded as fortuitous. For a given zero or favourable pressure gradient the typical channel-flow 'spot' tends to be wider in planform than the boundary-layer case anyway. The present adverse pressure gradient widens the boundary-layer 'spot', however, thus reducing the difference, but at the expense of creating extra disturbance growth near the spanwise edges which is quite different from the zero or favourable pressure gradient cases. The stronger (-) contribution near the sides of the 'spot' upstream may be connected (however fortuitously) with the extended side effects reported experimentally by Gostelow, Seifert, van Hest and their colleagues in the presence of adverse pressure gradients, even though we should emphasize immediately that the evolution theory here is not for the turbulent case. Moreover, tentatively, the sketch and flow features found in figure 4 are not unlike the experimental observations, for example, on spreading angles and amplitude maxima within an adverse pressure gradient 'spot', although here the adverse pressure gradient is still only mild.

\section{Nonlinear effects}

It is perhaps natural to consider planar nonlinear disturbances first, in view of the centreline results of $\S 4$. During stage 2 , the long timescale $\hat{t}\left(\equiv R e^{1 / 2} t\right)=\sigma^{-5} T$ comes into operation, in addition to $\hat{t}=\sigma^{-2} \tilde{t}$, so that $\partial / \partial t$ becomes $R e^{1 / 2} \sigma^{2}\left(\partial / \partial \tilde{t}+\sigma^{3} \partial / \partial T\right)$, and the streamwise dependence is on $\tilde{x}, X$ where $x-x_{1}=R e^{-1 / 2} \sigma^{-4} X$. In stage 2, 
however, the remaining rapid variation, i.e. in $\tilde{x}, \tilde{t}$, is solely in terms of factors $C, S$, effectively $\cos \Theta$, $\sin \Theta$ as implied by (3.10)ff, satisfying

$$
\partial_{x}(C, S)=R e^{1 / 2} \sigma F(-S, C), \partial_{t}(C, S)=R e^{1 / 2} \sigma^{2} G(-S, C) .
$$

Here, $F, G$ are functions of $X, T$ to be determined below, although we may anticipate them to be $\frac{1}{2} \eta,-\frac{1}{4} \eta^{2}$, respectively, from $\partial \Theta / \partial \tilde{x}, \partial \Theta / \partial \tilde{t}$ where $\Theta=\tilde{x}^{2} /(4 \tilde{t})-\frac{1}{4} \pi$ from stage 1 (e.g. see (3.10)) and $\eta$, which is of order unity, now stands for $X / T$. Moreover, the (planar) disturbance amplitude is taken to be as in (3.20) and so nonlinear influences are expected to appear in the leading critical layer, the continuation of layer (ii) from the previous section, while outside of that, linear dynamics is expected to apply.

\subsection{Majority of the flow}

The flow structure in the lateral direction is mainly as before, with three tiers I-III, but essentially supplemented by the leading critical layer (ii) and the trailing critical layer (iv). Tier I now has, with $\hat{\Delta}\left(\equiv \sigma^{1 / 2} \Delta\right)=\sigma^{4}$,

$$
\begin{aligned}
u & =\sigma Y+\sigma^{3} \bar{\lambda}_{1} Y+\sigma^{4} u_{4}(Y)+\hat{\Delta}\left[\tilde{u}_{0}+\cdots+\sigma^{3} \tilde{u}_{3} \cdots+\sigma^{6} \widetilde{u}_{6}+\cdots\right], \\
\psi & =\operatorname{Re}^{-1 / 2}\left\{\frac{1}{2} \sigma^{2} Y^{2}+\cdots+\hat{\Delta}\left[\sigma \tilde{\psi}_{0}+\cdots+\sigma^{4} \tilde{\psi}_{3} \cdots+\sigma^{7} \tilde{\psi}_{6},+\cdots\right]\right\}, \\
p & =\bar{p}(0)+\frac{1}{2} \bar{p}^{\prime \prime}(0) \bar{x}_{1}^{2} \sigma^{4}+\cdots+\hat{\Delta}\left[\sigma \tilde{p}_{0}+\cdots+\sigma^{4} \tilde{p}_{3} \cdots+\sigma^{7} \widetilde{p}_{6}+\cdots\right] .
\end{aligned}
$$

The continuity equation therefore yields $\tilde{u}_{n}=\partial \tilde{\psi}_{n} / \partial Y$, the $x$-momentum balance becomes

$$
\left[\mathscr{L}\left(\tilde{u}_{0}, \tilde{\psi}_{0}\right) \equiv\right] \tilde{u}_{0 \tilde{t}}+Y \tilde{u}_{0 \tilde{x}}-\tilde{\psi}_{0 \tilde{x}}=-\tilde{p}_{0 \tilde{x}}
$$

at leading order in the disturbance, and the $y$-momentum balance shows $\tilde{p}_{0-3}$ to be independent of $Y$. The solution satisfying (5.3a) here is

$$
\left[\tilde{u}_{0}, \tilde{\psi}_{0}, \tilde{p}_{0}\right]=\left[a_{0}, Y a_{0}, \pi_{0}\right] C, \text { with }-G a_{0}=F \pi_{0},
$$

and the scaled velocity and pressure amplitudes $a_{0}, \pi_{0}$ are functions of $X, T$ to be determined. The next $x$-momentum balance of significance occurs at relative order $\sigma^{3}$, namely

$$
\mathscr{L}\left(\tilde{u}_{3}, \tilde{\psi}_{3}\right)+C a_{0 T}+\left(u_{4}+\tilde{u}_{0}\right) \tilde{u}_{0 \tilde{x}}-\tilde{\psi}_{0 \tilde{x}}\left(u_{4 Y}+\tilde{u}_{0 Y}\right)=-\tilde{p}_{3 \tilde{x}}-C \pi_{0 X},
$$

after cancellation of two $Y C \partial a_{0} / \partial X$ terms. The nonlinear contributions in $\tilde{u}_{0} \partial \tilde{u}_{0} / \partial \tilde{x}$, $\left(\partial \tilde{\psi}_{0} / \partial \tilde{x}\right)\left(\partial \tilde{u}_{0} / \partial Y\right)(=0)$ are passive at this level, forcing second harmonics and meanflow corrections to arise. So the solution has the form

$$
\begin{gathered}
\left(\tilde{\psi}_{3}, \tilde{p}_{3}\right)=S\left(\tilde{\psi}_{31}, \tilde{p}_{31}\right)+C\left(\tilde{\psi}_{32}, \tilde{p}_{32}\right)+\left(C^{2}-S^{2}\right)\left(\tilde{\psi}_{33}, \tilde{p}_{33}\right)+\left(\widetilde{\psi}_{3 m}, \widetilde{p}_{3 m}\right), \\
\left\{(Y F+G) \partial_{Y}-F\right\}\left(-\tilde{\psi}_{31}, \tilde{\psi}_{32}\right)+F\left(-\tilde{p}_{31}, \tilde{p}_{32}\right)=\left(a_{0 T}+\pi_{0 X},\left[Y u_{4}^{\prime}-u_{4}\right] F a_{0}\right),
\end{gathered}
$$

with the mean-flow correction terms remaining undetermined at this order. For the two components in $C, S,(5.4 d)$ yields

$$
\left(\tilde{\psi}_{32}\right)_{Y Y}=Y u_{4}^{\prime \prime} a_{0} /(Y+G / F),
$$

and, hence, a critical level (see layer (ii) below) at $Y=-G / F \equiv \tilde{Y}_{g}$, inducing a jump (j) from which the local behaviour

$$
\left(\tilde{\psi}_{32}\right)_{Y} \sim \tilde{Y}_{g} u_{4 g}^{\prime \prime} a_{0} \ln \left|Y-\tilde{Y}_{g}\right|, \quad\left[\tilde{\psi}_{31}\right] \sim-\tilde{Y}_{g} u_{4 g}^{\prime \prime} a_{0} j\left(Y-\tilde{Y}_{g}\right),
$$


is obtained near $Y=\tilde{Y}_{g}$, with $u_{4 g}^{\prime \prime}$ denoting $u_{4}^{\prime \prime}$ evaluated at $\tilde{Y}_{g}$. Here, $\left[\tilde{\psi}_{31}\right]$ stands for the difference between $\tilde{\psi}_{31}$ as $Y^{\prime g} \rightarrow \tilde{Y}_{g}+, \tilde{Y}_{g}-$, and the velocity-like jump $j$ is to be found below from analysis of the moving critical layer (ii). The forced contribution $(5.5 c)$ is in addition to that implied directly by $(5.4 c)$,

$$
\tilde{\psi}_{31}=a_{31} Y \quad \text { with } G a_{31}+F \tilde{p}_{31}=-a_{0 T}-\pi_{0 X},
$$

for $Y<\tilde{Y}_{g}$, in view of the tangential-flow constraint at the wall.

Tier II is described by

$$
\begin{aligned}
u & =\bar{u}_{0}(\bar{y})+\sigma^{2} \bar{u}_{2}(\bar{y})+\cdots+\hat{\Delta}\left[u^{(0)}+\sigma u^{(1)}+\cdots\right], \\
\psi & =\operatorname{Re}^{-1 / 2}\left\{\bar{\psi}_{0}(\bar{y})+\sigma^{2} \bar{\psi}_{2}(\bar{y})+\cdots+\hat{\Delta}\left[\psi^{(0)}+\sigma \psi^{(1)}+\cdots\right]\right\}, \\
p & =\cdots+\hat{\Delta}\left[\sigma p^{(0)}+\cdots\right],
\end{aligned}
$$

with only $p^{(0)}$ being independent of $\bar{y}$. Here, the leading-order disturbance solution is found to be

$$
\left[\psi^{(0)}, u^{(0)}, v^{(0)}, p^{(0)}\right]=\left[C a_{0} \bar{u}_{0}, C a_{0} \bar{u}_{0}^{\prime}, F S a_{0} \bar{u}_{0}, C \pi_{0}\right],
$$

corresponding to the usual displacement form, for example as in (3.5a). At higher order, as further inertial forces and non-zero pressure gradients enter play,

$$
\begin{aligned}
\psi^{(1)}= & C\left\{-\tilde{Y}_{g} a_{0}-\pi_{0} \bar{u}_{0} I_{1}+a_{1} \bar{u}_{0}\right\}, \\
\psi_{\tilde{x}}^{(2)}= & S\left\{-F a_{0} \bar{u}_{2}-G\left[2 \pi_{0} \bar{u}_{0} I_{2}-\pi_{0} I_{1}+a_{1}\right]\right. \\
& \left.-F \bar{u}_{0}\left[p_{\tilde{x}}^{(1)}(\bar{y}=0) I_{1}-a_{2}-F^{2} a_{0} I_{3}\right]\right\},
\end{aligned}
$$

remain in phase with $\psi^{(0)}$, whereas $\psi^{(3)}$ has an out-of-phase component $S \hat{a}_{31} \bar{u}_{0}$, in which

$$
\hat{a}_{31} \equiv a_{31}-\tilde{Y}_{g} u_{4 g}^{\prime \prime} j a_{0} .
$$

In (5.8b) $I_{2}, I_{3}$ denote the $P . V$. integrals of $\bar{u}_{0}^{-3}, I_{1} \bar{u}_{0}^{-2}$ with respect to $\bar{y}$, in turn. Tier III, just outside the boundary layer, consists of potential flow essentially as in $\$ \S 2$ and 3 and yields the pressure-displacement laws

$$
\pi_{0}=F a_{0}, \quad \tilde{p}_{31}=F \hat{a}_{31}+\partial a_{0} / \partial X,
$$

from matching with the solution of tier II. The dominant pressure disturbance here is $\Delta \sigma \pi_{0} C \exp (-F \overline{\bar{y}})$ with $F$ being assumed positive, while $\tilde{p}_{31}$ is the higher-order pressure disturbance in $(5.4 b)$.

Combining $(5.10 a)$ with $(5.3 b)$, we therefore obtain the primary relation

$$
G=-F^{2},
$$

or

$$
\Theta_{\tilde{t}}=-\Theta_{\tilde{x}}^{2},
$$

(see also Whitham 1974). This is consistent with the simple form $\tilde{x}^{2} /(4 \tilde{t})-\frac{1}{4} \pi$ for $\Theta$ as anticipated near the start of this section. It is worth noting in passing that $(5.11 b)$ gives the inviscid Burgers equation $\partial \tilde{\phi} / \partial \tilde{t}+2 \tilde{\phi} \partial \tilde{\phi} / \partial \tilde{x}=0$ for $\tilde{\phi} \equiv \partial \Theta / \partial \tilde{x}$, allowing discontinuities to form at finite time $\tilde{t}$ under suitable disturbed initial conditions; a similar point is made by Gajjar \& Smith (1985). However, we take it that $\Theta$ is described by the simple form above, leaving $F=\frac{1}{2} \eta$ and $G=-\frac{1}{4} \eta^{2}$, and in consequence $\tilde{Y}_{g}=Y_{g}$. The next relation of concern comes from the pressure match 
in $(5.10 b)$, or from matching terms in $S$ between tiers I and II, which yields

$$
F^{-1}\left(a_{0 T}+\pi_{0 X}\right)=Y_{g}^{2} u_{4 g}^{\prime \prime} j a_{0}-a_{0 X}
$$

or, upon substitution for $F, G, Y_{g}, u_{4}^{\prime \prime}, \pi_{0}$, the scaled velocity amplitude equation

$$
\left[\partial_{T}+\eta \partial_{X}\right]\left(T^{1 / 2} a_{0}\right)=\frac{j \widetilde{g} \eta^{3}}{8}\left(1-\frac{3 \kappa \eta^{2}}{\widetilde{g}}\right)\left(T^{1 / 2} a_{0}\right)
$$

for $a_{0}(X, T)$ in the current stage 2 .

For small times $T$, the jump $j$ is expected to be nearly equal to $\pi$ and then the growth rate from $(5.12 b)$ is observed to agree with that implied in (2.14) and (3.19). Thus, there is agreement with the Rayleigh result of $\S 2$ as well as matching with the solution of the earlier transient stage 1 . Moreover, the grouping $\left(T^{1 / 2} a_{0}\right)$ here indicates a merging with the earlier amplitude behaviour (3.10) and (3.19). The value $\pi$ for $j$ also covers the case of comparatively small, linear, disturbances where $\hat{\Delta}$ is reduced, as in $\S \S 4$ and 5.3.

\subsection{The leading critical layer}

At order-one times $T$, and for nonlinear disturbances in general, the jump $j$ is fixed as follows by the properties in the moving critical layer (ii), wherein $\bar{y}=\sigma Y_{g}+\sigma^{5 / 2} Y_{1}$ with $Y_{1}$ of $O(1)$ and $Y_{g}=\frac{1}{2} \eta\left(=\tilde{Y}_{g}\right)$ from the forms of $F, G$. In (ii),

$$
\begin{aligned}
u & =\sigma Y_{g}+\sigma^{5 / 2} Y_{1}+\sigma^{3} \bar{\lambda}_{1} Y_{g}+\sigma^{4} u_{4 g} \ldots+\hat{\Delta}\left[C a_{0}+\sigma^{3} U_{1}+\sigma^{9 / 2} U_{2}+\cdots\right], \\
\psi & =\operatorname{Re}^{-1 / 2}\left\{\frac{1}{2} \sigma^{2} Y_{g}^{2}+\cdots+\hat{\Delta}\left[\sigma C a_{0} Y_{g}+\sigma^{5 / 2} C a_{0} Y_{1}+\sigma^{4} a_{2}+\sigma^{11 / 2} \cdots\right]\right\}, \\
p & =\cdots+\hat{\Delta}\left[\sigma C \pi_{0}+\sigma^{4} \widetilde{p}_{3}+\cdots+\sigma^{11 / 2} P_{2}+\sigma^{13 / 2} P_{3}+\cdots\right],
\end{aligned}
$$

where $a_{2}$ denotes $\widetilde{\psi}_{3}$ evaluated at $Y=Y_{g}, P_{3}$ is the leading $Y$-dependent pressure term and, to match with the solution in tier I,

$$
\left.U_{1} \sim Y_{g} u_{4 g}^{\prime \prime} C a_{0}\left(\ln \left|Y_{1}\right|+1\right)+\left\{S a_{31}+C \psi_{32}^{\prime} \text { (F.P., } Y=Y_{g}\right)\right\}+\cdots+\text { (zero or jump) }
$$

at large $\left|Y_{1}\right|$. Also, the lateral velocity is given by

$$
\begin{aligned}
v= & \hat{\Delta}\left[\sigma^{2}\left(F S a_{0} Y_{g}\right)+\sigma^{7 / 2}\left(F S a_{0} Y_{1}\right)+\sigma^{5}\left\{-C\left(a_{0} Y_{g}\right)_{X}+a_{2 \tilde{x}}+\frac{C a_{0}}{2 T}\right\}\right. \\
& \left.+\sigma^{13 / 2}\left(-\Psi_{1 \tilde{x}}-C a_{0 X} Y_{1}\right)+\sigma^{8}\left(\frac{\Psi_{1 Y_{1}}}{2 T}-a_{2 X}\right)+\cdots\right]
\end{aligned}
$$

from $(5.13 b)$. The governing equations here, in succession, confirm the balance $(5.3 b)$ and then the rapid dependence on $\tilde{x}, \tilde{t}$ in (5.1), before producing the vorticity or shear equation for $\tau_{1} \equiv \partial U_{1} / \partial Y_{1}$,

$$
Y_{1} \frac{\partial \tau_{1}}{\partial \tilde{x}}+\left(F^{2} S a_{0}+\frac{X}{4 T^{2}}\right) \frac{\partial \tau_{1}}{\partial Y_{1}}=-F^{2} u_{4 g}^{\prime \prime} a_{0} S .
$$

At large $\left|Y_{1}\right|$, this is subject to the condition $(5.14 a)$, the leading term of which is consistent with a balance between the first and last terms in $(5.14 c)$. The quantities $F^{2} a_{0}, X /\left(4 T^{2}\right)$ and $u_{4 g}^{\prime \prime}$ in $(5.14 c)$ act as parameters in determining the rapid variation of $\tau_{1}$ with $\tilde{x}$ (and $Y_{1}$ ), in terms of $S, C$ and powers thereof, and the induced jump $j$. 
At this stage, the main particle paths within (ii) are assumed to remain open, corresponding to $X a_{0}<1$ from the $\partial \tau_{1} / \partial Y_{1}$ coefficient in $(5.14 c)$. The present nonlinear group critical layer is of the moving type, e.g. Gajjar \& Smith (1985), rather than the non-equilibrium type, e.g. Hickernell (1984), Goldstein et al. (1987) and others, the movement being defined by its position $Y=X /(2 T)\left[=Y_{g}=\frac{1}{2} \eta=F\right]$ which represents an increasing departure from the wall and which, in a sense, marks the leading edge of the disturbance fluctuation as it travels downstream.

Nonlinearity is present because of the $F^{2} S a_{0}$ part. As a check, at small $T$, that part becomes small relative to the $X /\left(4 T^{2}\right)$ linear part, by a factor $T^{1 / 2}$, in keeping with the earlier stage 1. Again, for small velocity amplitudes $\left|a_{0}\right|$ the required solution of $(5.14 a, c)$ is

$$
\tau_{1}=\operatorname{Im}\left[\exp \left(\mathrm{i}\left(\Theta-\tilde{\gamma} Y_{1}^{2}\right)\right) \frac{4 T^{2} R}{X}\left\{\int_{0}^{Y_{1}} \exp \left(\mathrm{i} \tilde{\gamma} \bar{Y}_{1}^{2}\right) \mathrm{d} \bar{Y}_{1}+\frac{1}{2}\left(\frac{\pi}{\tilde{\gamma}}\right)^{1 / 2} \exp (\mathrm{i} \pi / 4)\right\}\right]
$$

where $R S$ is the right-hand side of $(5.14 c), \tilde{\gamma} \equiv 2 F T^{2} / X$ and $\tau_{1} \rightarrow 0$ as $Y_{1} \rightarrow-\infty$. Since the integral in (5.15) is odd in $Y_{1}$, the velocity jump contribution from the integral of $\tau_{1}$ over all $Y_{1}$ is then $-\pi F u_{4 g}^{\prime \prime} a_{0} S$, giving $j$ equal to $\pi$ as expected. The linear case (5.15) also shows that fast waves persist in the vorticity $\tau_{1}$ at large positive $Y_{1}$, as implied by $\S 3$. These waves are cancelled out in the second critical layer, effectively (iv), which is associated with the group velocity and is sited at a larger $Y$ position, marking the trailing edge of the fluctuating vorticity disturbance. Figure $5(a)$ gives a summary sketch in the $(X, T)$-plane to highlight the leading and trailing edges of the 'spot' as far as the present small or non-small scaled vorticity waves are concerned. For non-small amplitudes, the required solution of $(5.14 a, c)$ is given by Smith \& Bodonyi (1987).

\subsection{Linear and nonlinear solutions}

The nonlinear stage-2 problem overall is to solve, in the $(\eta, T)$ frame for convenience,

$$
\frac{\partial A}{\partial T}=j(m) \frac{\widetilde{g} \eta^{3}}{8}\left(1-\frac{3 \kappa \eta^{2}}{\widetilde{g}}\right) A
$$

for $A(\eta, T)$, from (5.12b) with $A \equiv T^{1 / 2} a_{0}$, but with the amplitude-dependent jump $j$ being the function of $m \equiv\left(T^{1 / 2} A \eta\right)^{-1}$ which is given in Smith \& Bodonyi's (1987) figure $1(b)$ and equation (2.16) (written there as $|\phi|$ being a function of their parameter $\mu$ ). The function $j(m)$ may actually be modelled, or approximated fairly closely, by the formula

$$
j(m)=\pi\left\{1+\frac{1}{4 m^{2}}+\frac{5}{m^{4}}\left(\frac{1}{\pi}-\frac{1}{4}\right)\right\},
$$

from the above paper, although the latter notes the presence of an $(m-1) \ln (m-1)$ behaviour near $m=1+$. It is borne in mind also that the theory so far holds for $m>1\left[X a_{0}<1\right]$, because of the change to a closed streamline pattern in (ii) if $m$ falls below 1, i.e. if the amplitude $A$ exceeds $\eta^{-1} T^{-1 / 2}$ at any $\eta, T$ value. The initial condition is

$$
A=v_{1} e^{-q \eta / 2} \quad \text { at } T=0+
$$

for the main example of $\S 3$, where $v_{1}$ is a constant which may be taken as positive without loss of generality. Along any given line $\eta(=X / T)=$ constant, $(5.16 a)$ acts 
192

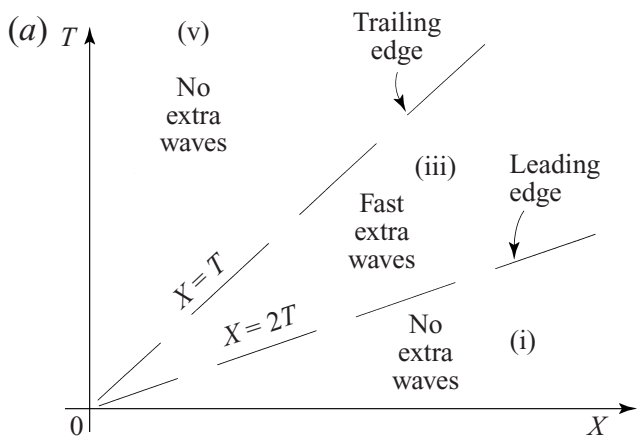

(c)
F. T. Smith and S. N. Timoshin
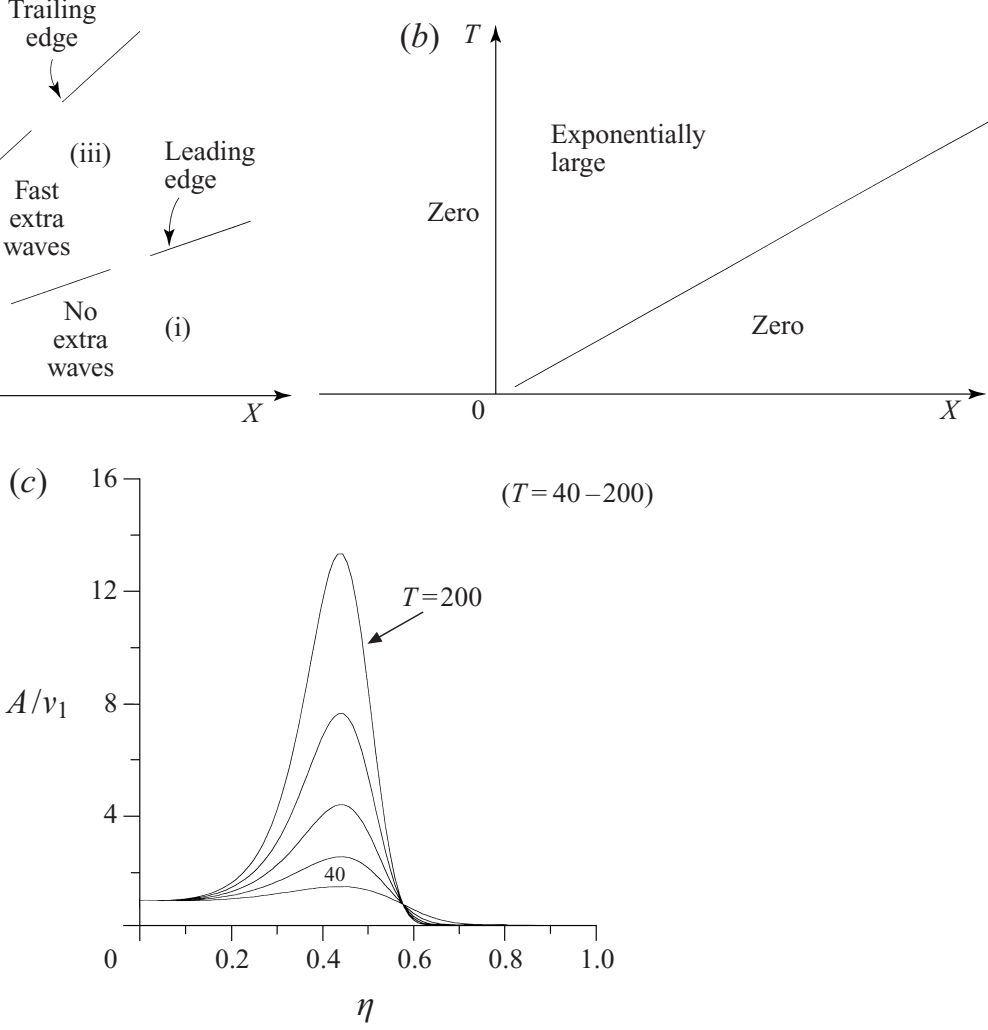

FiguRE 5. $(a, b)$ Leading edge and trailing edge of planar linear 'spot', between which $(a)$ the scaled vorticity and $(b)$ the scaled velocity fluctuation $A$ concentrates at large time $T(\$ 5.3)$. (c) Solution (5.17).

as a nonlinear ordinary differential equation controlling $A$ for positive $T$, given the starting form $(5.16 c)$.

For linear disturbances $\left(v_{1}\right.$ small $), j \approx \pi$ and the velocity amplitude solution is

$$
A=B(\eta) \exp \left[\pi \frac{\widetilde{g} \eta^{3}}{8}\left(1-\frac{3 \kappa \eta^{2}}{\widetilde{g}}\right) T\right],
$$

for all $T>0$, from (5.16a). The function of integration, $B$, then follows from (5.16c) as $v_{1} \exp \left(-\frac{1}{2} q \eta\right)$, matching with stage 1 at small $T$. At large $T$, the solution $A$ becomes exponentially large for $0<\eta<(\widetilde{g} / 3 \kappa)^{1 / 2}$, i.e. in the domain $0<X<(\widetilde{g} / 3 \kappa)^{1 / 2} T$, and exponentially small outside, in line with the results of $\S 4$, e.g. see (4.7). A wedge of velocity amplitude growth is thus produced in the domain above, as summarized in figure $5(b)$ (which contrasts with the vorticity diagram in figure $5(a)$ ) and shown numerically for a specific example in figure 5(c) at increasing times.

For nonlinear disturbances $\left(v_{1} \sim 1\right)$, numerical solutions of $(5.16 a-c)$ are presented in figure 6 . The figure shows the evolution of the scaled velocity amplitude $A$ and the phase property $j$ for three different initial amplitudes. The two higher initial amplitudes lead eventually to the upper limitation on amplitude being encountered within the time range of the computations. In general, at early times, the response is similar to (5.17), but eventually the upper limit $A \rightarrow \eta^{-1} T^{-1 / 2}(m \rightarrow 1+)$ on the velocity amplitude is attained at a finite positive $(X, T)$ value as indicated. That heralds a localized breakdown of the nonlinear theory behind $(5.16 a, b)$. 
(a)

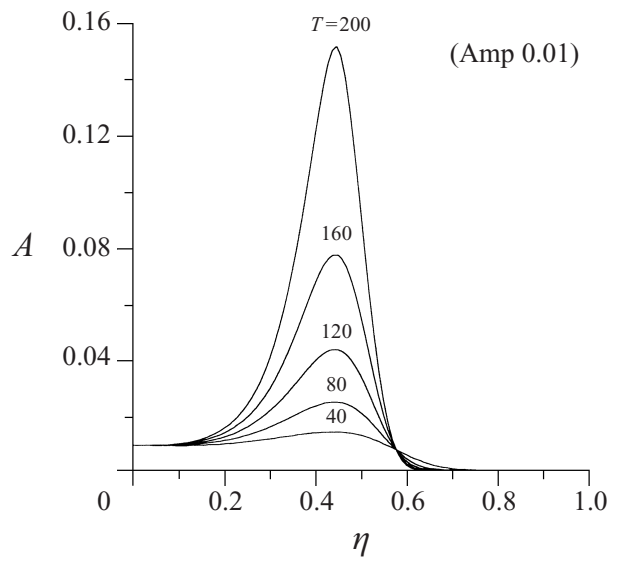

(b)

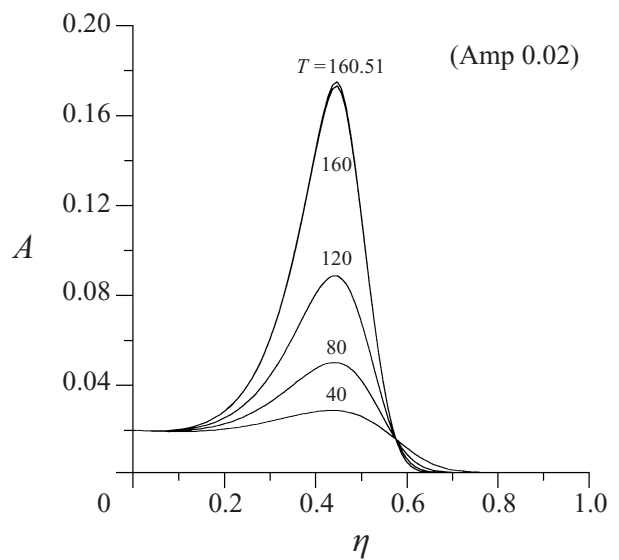

(c)

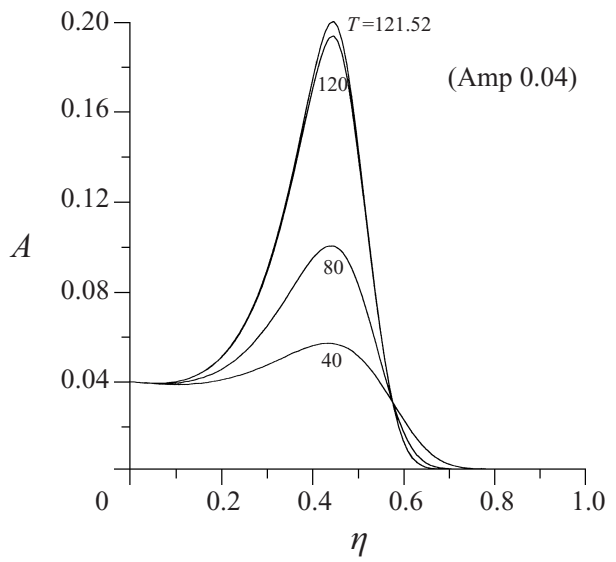

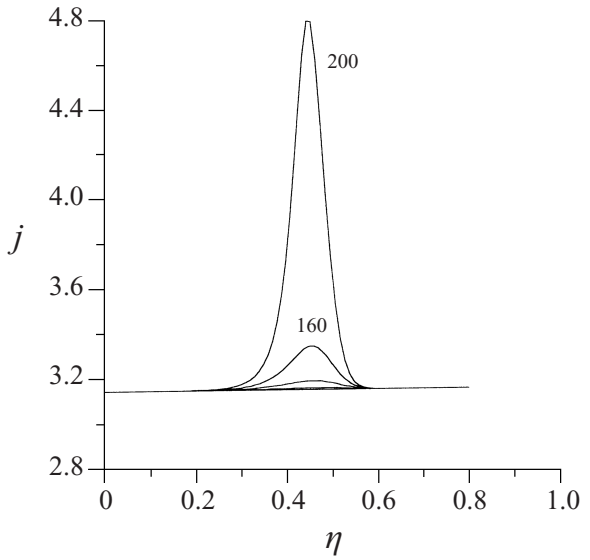
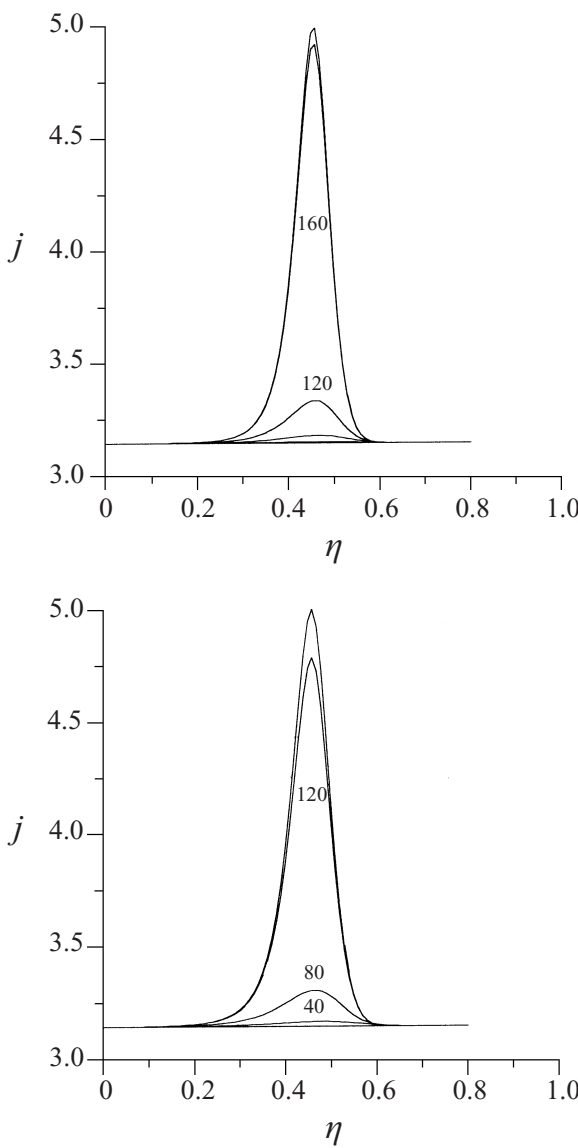

FIGURE 6. Nonlinear 'spot' solutions of $(5.16 a-c)$ for initial amplitudes $(a) 0.01,(b) 0.02,(c) 0.04$, showing amplitude $A$ and phase function $j$. Note the upper limitation $A=\eta^{-1} T^{-1 / 2}(\S 5.3)$, which is reached in cases $(b),(c)$ at the times indicated, but not in case $(a)$ for the time range shown.

\section{Nonlinear effect on the mean flow}

The nonlinear behaviour of the fluctuating component in $\S 5$ has an effect on the mean flow throughout tiers I-III of the 'spot', as well as interactively inside the critical layer (ii). 
In tier I, both the velocity and the vorticity of the mean-flow correction experience jumps across the leading critical layer (ii). The mean velocity jump $u_{J m}(X, T)$ in scaled form comes from $(5.14 c)$ and is

$$
u_{J m}=\frac{1}{4} a_{0}^{2} T \eta^{2} u_{4 g}^{\prime \prime} j
$$

with $j, a_{0}$ determined as in $\S 5.3$. The induced mean-flow correction first appears in the terms with subscript $3 m$ in $(5.4 b)$, independent of the fast variation $C, S$. The governing equations for these terms are obtained at higher order, from the balance

$$
\begin{aligned}
\mathscr{L}\left(\widetilde{u}_{6}, \widetilde{\psi}_{6}\right) & +\widetilde{u}_{3 T}+\widetilde{u}_{3} \widetilde{u}_{0 \widetilde{x}}+\left(\widetilde{u}_{4}+\widetilde{u}_{0}\right)\left(\widetilde{u}_{3 \widetilde{x}}+\widetilde{u}_{0 X}\right) \\
& +Y \widetilde{u}_{3 X}-\widetilde{\psi}_{3 X}-\left(\widetilde{\psi}_{3 \tilde{x}}+\widetilde{\psi}_{0 X}\right)\left(\widetilde{u}_{4}^{\prime}+\widetilde{u}_{0 Y}\right)-\widetilde{\psi}_{0 \widetilde{X}} \widetilde{u}_{3 Y}=-\widetilde{p}_{6 \widetilde{x}}-\widetilde{p}_{3 X},
\end{aligned}
$$

which, as a check, holds strictly provided that $\sigma^{10} \gg R e^{-1 / 2}$, so that viscous forces remain negligible. Also, $\widetilde{p}_{3}$ is independent of $Y$. Hence, the contributions with slower streamwise and temporal dependence yield the momentum equation

$$
\left(\partial_{T}+Y \partial_{X}\right) \widetilde{u}_{3 m}-\partial_{X} \widetilde{\psi}_{3 m}+\frac{1}{2}\left(a_{0} a_{0 X}+Y a_{0} F \partial_{Y} \widetilde{u}_{31}\right)=-\widetilde{p}_{3 m X}
$$

for the mean-flow correction, along with $\widetilde{u}_{3 m}=\partial \tilde{\psi}_{3 m} / \partial Y, \partial \widetilde{p}_{3 m} / \partial Y=0$ and

$$
\widetilde{\psi}_{3 m}=0 \quad \text { at } Y=0+.
$$

From (5.4c), however, $\partial \widetilde{u}_{31} / \partial Y$ is identically zero, and so $(6.3 a)$ gives $(\partial / \partial T+$ $Y \partial / \partial X) \widetilde{\tau}_{3 m}=0$ for the vorticity $\widetilde{\tau}_{3 m} \equiv \partial \widetilde{u}_{3 m} / \partial Y$. Therefore,

$$
\tilde{\tau}_{3 m}=f_{3 m}(X-Y T, Y) \text {, }
$$

with the function $f_{3 m}$ to be found below for the areas (i), (iii) and (v) of tier I. Zero initial vorticity $\tilde{\tau}_{3 m}$ is assumed.

Area (i) is ahead of or underhangs the leading edge (ii) of the 'spot' and so we impose $\widetilde{\tau}_{3 m} \equiv 0$ in (i), for $0<Y<X /(2 T)$. Across the leading, lower critical layer (ii) a jump $\tau_{J m}(X, T)$ in the scaled vorticity $\widetilde{\tau}_{3 m}$ is supposed, this value therefore holding at the lower edge $(Y=X /(2 T)+)$ of area (iii) and acting to fix $f_{3 m}$ in (6.4) for area (iii). Thus

$$
f_{3 m}(X-Y T, Y)=\tau_{J m}\left(2[1-a] X,\left[\frac{1-a}{a}\right] T\right),
$$

where $\frac{1}{2}<a<1, Y=a X / T$ in (iii), so that $f_{3 m}$ is determined once values of $\tau_{J m}$ at earlier positive times and positive $X$ stations are found (below). The same argument shows that $f_{3 m}$ is zero in area (v), and indeed $f_{3 m}$ tends to zero at the upper edge $Y=X / T-$ of (iii). This upper edge, which marks the position of the trailing critical layer, corresponds to $a$ being unity in the right-hand side of (6.5) and, hence, yields zero mean vorticity alteration $f_{3 m}$ since the initial correction is zero. The same feature follows directly from the form of the operator in the governing equation $(6.3 a)$ for the mean flow correction, confirming that the upper edge of the mean vorticity alteration departs from the wall, as depicted in figure 7(a). The rate of departure of the upper (trailing) edge is twice that of the lower (leading) because of the respective $Y$ positions $X / T, X /(2 T)$ of the two critical layers.

No jumps occur in the corresponding pressure $\widetilde{p}_{3 m}(X, T)$, across (ii). Further, matching outwards through tiers II and III shows that $\widetilde{p}_{3 m}$ must be identically zero, because of the implied longer lengthscale of the mean-flow correction compared with that of the fluctuating components. It follows that in area (i) $\widetilde{u}_{3 m}$ is independent of $Y$, so that $\widetilde{u}_{3 m}=\widetilde{\widetilde{u}}_{3 m}(X, T)$ say, and, hence, $\widetilde{\psi}_{3 m}=Y \widetilde{\widetilde{u}}_{3 m}$ in view of $(6.3 b)$. So in (i), 
the momentum balance in $(6.3 a)$ leads to the relation

$$
\partial_{T}\left(\tilde{\tilde{u}}_{3 m}\right)=-\frac{1}{2} a_{0} a_{0 X}
$$

controlling the mean velocity solution, in particular, giving the induced mean slip velocity at the wall for positive $X$, thus representing a Benjamin-Ono-like mean flow effect (a nonlinear influence which enters simultaneously with that inside the leading layer (ii)). In area (iii), a similar integration of (6.4) in $Y$ with (6.5) leads to

$$
\widetilde{\psi}_{3 m}=\int_{X / 2 T}^{Y}\left\{\int_{X / 2 T}^{Y_{1}} f_{3 m}\left(X-Y_{2} T, Y_{2}\right) \mathrm{d} Y_{2}\right\} \mathrm{d} Y_{1}+Y \widetilde{\tilde{u}}_{3 m}+\left(Y-\frac{X}{2 T}\right) u_{J m}
$$

and, hence, to

$$
\frac{X}{4 T^{2}} \tau_{J m}=-\left[\left(u_{J m}\right)_{T}+\frac{\left(X u_{J m}\right)_{X}}{2 T}\right] .
$$

This controls the mean vorticity jump $\tau_{J m}$ in terms of the known velocity jump $u_{J m}$ given by (6.1). It can be shown that the relation $(6.7 b)$ is consistent with higher-order jump properties in the leading critical layer (ii).

The result of most immediate interest is (6.6), giving the induced mean wall slip under and ahead of the 'spot', subject to the initial condition $\widetilde{\widetilde{u}}_{3 m}=0$ at time $T=0+$ for all positive $X$. We notice that the time derivative in (6.6) is at a fixed position $X$, whereas that in $(5.16 a)$ for the fluctuation is (travelling) for fixed $\eta$. In a sense, this confirms that at a given streamwise position the main fluctuations sweep by, leaving mean-flow alterations behind.

The solution of (6.6) obtained numerically is presented in figure $7(b)$, for three different initial fluctuation amplitudes. The figure plots the temporal evolution of $T \widetilde{\widetilde{u}}_{3 m}$ versus the appropriate scaled streamwise coordinate $\eta(=X / T)$. At comparatively low input amplitudes, this mean-flow solution continues for a long way downstream, and for a relatively long time, developing an exponentially growing and expanding form in $\eta, T$ at large $T$. We observe also the similarity form holding at the start, for small $T$, in which $T \widetilde{\widetilde{u}}_{3 m}$ is a function of $\eta$, along with $T^{1 / 2} a_{0}$, this form being accommodated in the computations. At medium input amplitudes the nonlinear solution clearly terminates at a finite time, as indicated by the jump function attaining its maximum value (or $A$ its maximum). This termination time becomes shorter with increasing input and tends to zero as the input amplitude becomes relatively large; see further comments in $\S 7$. Also, in most cases, there is a pronounced expanding region where the induced mean velocity $\widetilde{\widetilde{u}}_{3 m}$ is negative, this region moving essentially with the 'spot' and supplementing the fixed region of positive and negative velocity values which trails behind. The above is for $X$ (and $T$ ) positive, whereas $f_{3 m}$, etc., like $\widetilde{\tilde{u}}_{3 m}$, are zero for $X$ negative behind the 'spot' trailing edge.

Behind the 'spot', in fact, the induced mean wall slip velocity is less in magnitude, its pressure being $\sigma^{11} p_{I}$ where,

$$
\begin{gathered}
p_{I}(X, T)=\frac{1}{\pi}(P . V .) \int_{0}^{\infty}\left(\widetilde{A}_{3 m}\right)_{\xi}(\xi, T) \frac{\mathrm{d} \xi}{X-\xi}, \\
\widetilde{A}_{3 m}=\int_{X /(2 T)}^{X / T} f_{3 m}(X-Y T, Y) \mathrm{d} Y+\widetilde{\tilde{u}}_{3 m}+u_{J m} .
\end{gathered}
$$

The induced displacement term in (6.8b) can be evaluated from (6.1), (6.5), (6.6), (6.7b) and then substituted into (6.8a) to fix the upstream wall pressure. See figures $7(c)$ and $7(d)$, which show the quantities involved. In particular, figure $7(c)$ shows the 
(a)
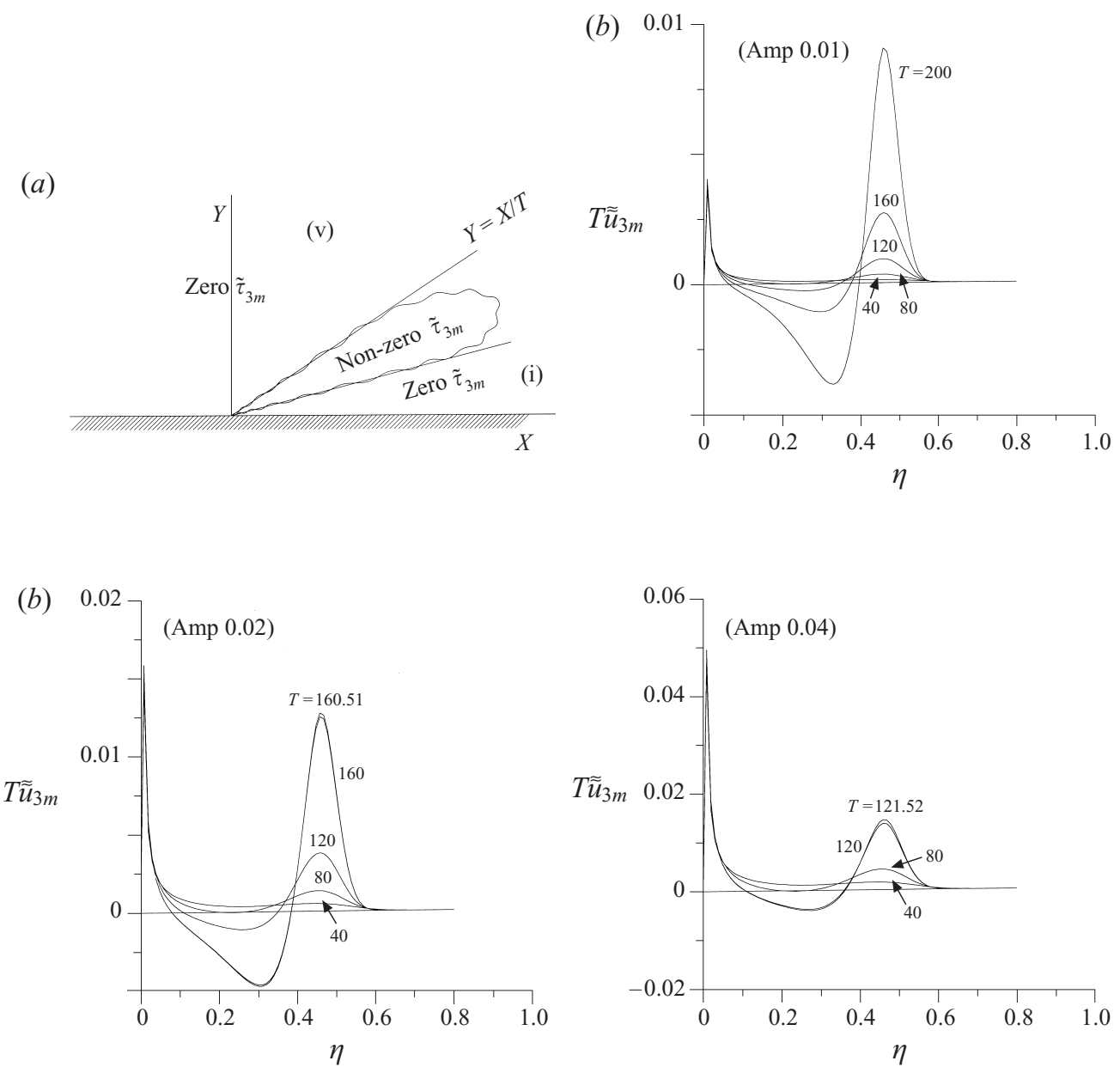

(c)

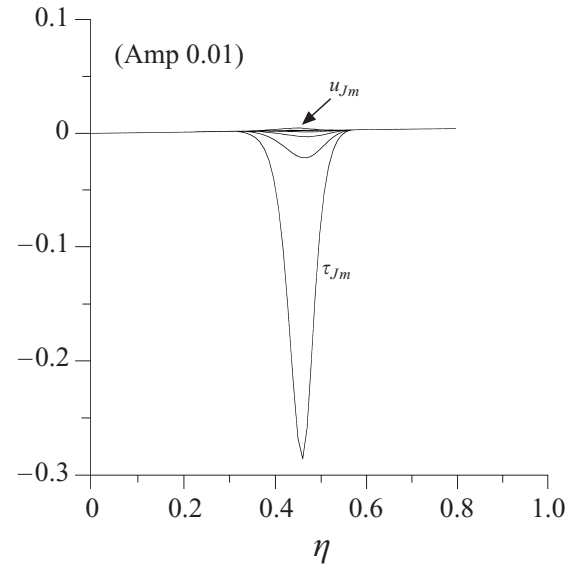

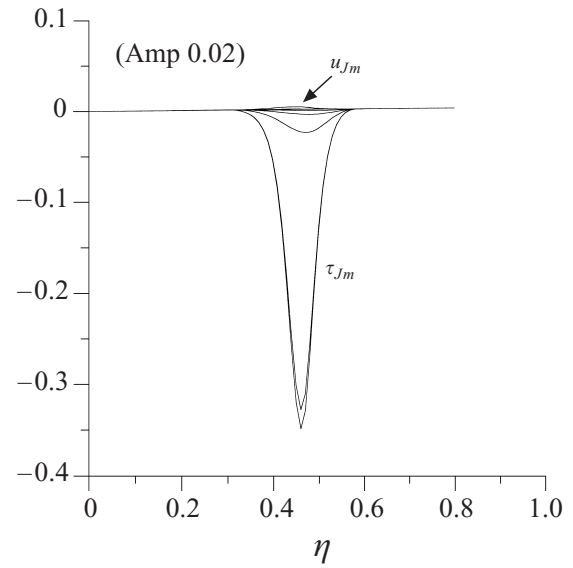

FigURE $7(a-c)$. For caption see facing page. 

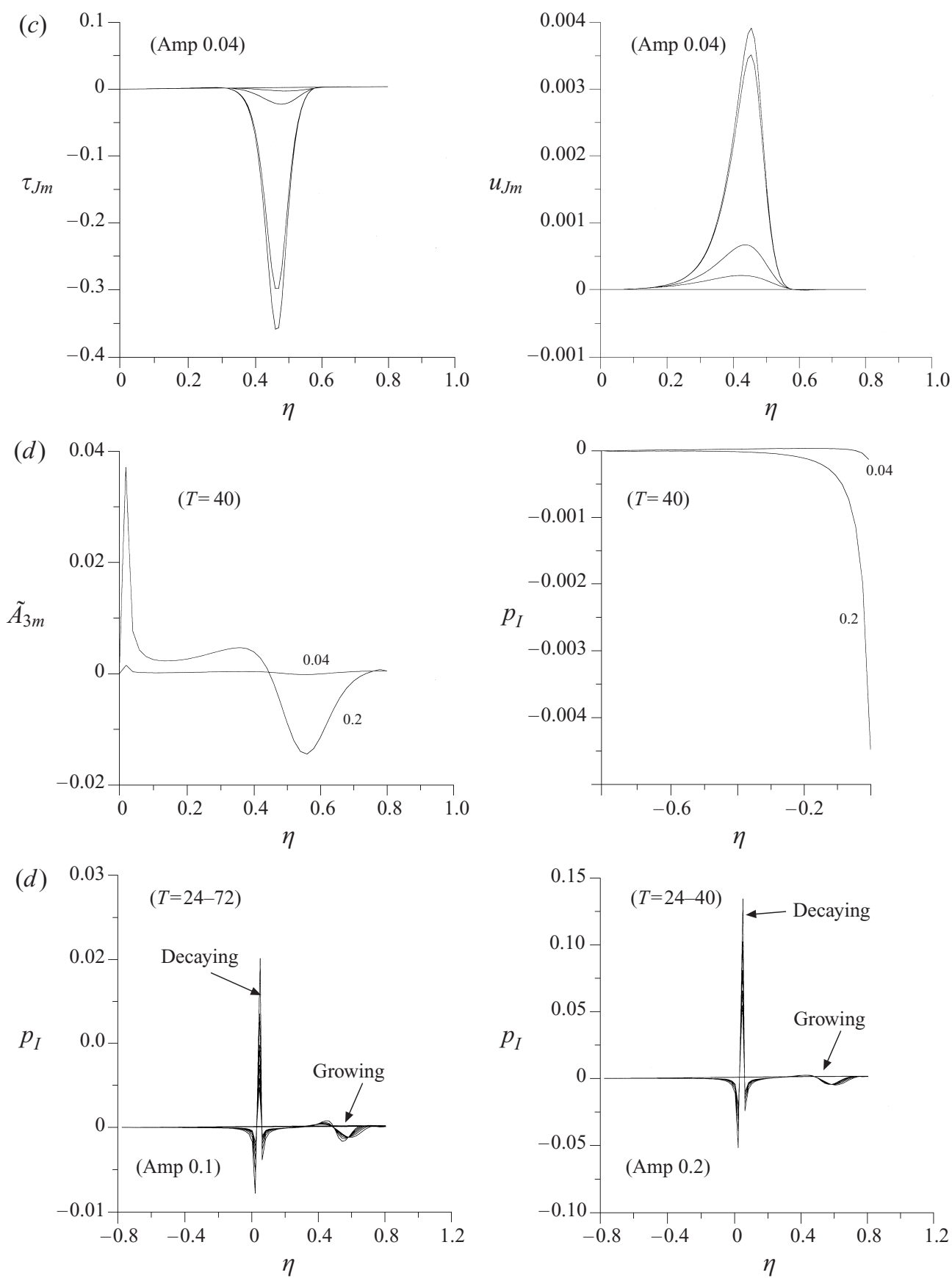

FiguRE 7. Mean-flow alterations $(\S 6)$. (a) The mean-flow structure, including the departures from the wall of the leading edge $(X /(2 T))$ and trailing edge $(X / T)$ of the mean vorticity distortion in the 'spot'. (b) Computed solutions for the mean velocity $\widetilde{\tilde{u}}_{3 m}$ produced ahead of/under the 'spot', from (6.6) with $a_{0}\left(=T^{-1 / 2} A\right)$ given as in figure 6 , for three different input amplitudes; termination times are shown for the two larger input amplitudes. (c) Mean shear $\tau_{J_{m}}$ and extra velocity $u_{J_{m}}$. (d) The induced mean displacement and pressure behind and within the 'spot'. 
development of the mean jump properties $u_{J m}, \tau_{J m}$ for the same three input amplitudes as in figure $7(b)$. Figure $7(d)$ gives samples of the mean pressure and displacement $p_{I}, \tilde{A}_{3 m}$ for one of the input amplitudes above and for two higher amplitudes of 0.1 and 0.2 , in order to clarify the temporal evolution. Low input amplitudes lead to a quasi-similarity exponential form at large times, whereas medium or high input produces earlier termination via increased nonlinear effects throughout. Zones of positive and negative induced pressure both appear, indicating trends towards and away from mean flow separation, behind the main 'spot'. On the other hand, these pressures decay with time behind the main 'spot' and, within it, their corresponding slip velocities are less in magnitude than those of (6.6).

\section{Final comments (including non-parallel-flow effects)}

\subsection{General points}

This work has been on linear and nonlinear unsteady disturbances introduced into a laminar boundary layer mostly at or near the onset of an adverse pressure gradient, leading to 'spot' growth downstream at later times. In contrast with recent studies of nonlinear onset evolutions by Savin et al. (1999) for surface-roughness transitions, where the effective phase-speed response is constant, and by Smith et al. (2000) for near-wake transitions, where instead the effective frequency response is constant, in the current setting the effective phase velocity plays a central role in the evolution of the fluctuating disturbances, along with its corresponding critical layer which travels across the flow and which helps form the leading edge of the 'spot'. It should be stressed that, on the other hand, the alteration of the mean flow vorticity takes place in the region lying between the lower critical layer (leading edge) and the upper critical layer (acting as the 'spot' vorticity trailing edge) as both layers leave the wall downstream at later scaled times.

The theory in $\$ \S 2-4$ covers planar or three-dimensional 'spots', while that on nonlinearity in $\S \S 5$ and 6 is for the planar case as a first step, given the dominance of planar growth in the form of the (+) contributions of $\S 4$ along the 'spot' centreline. It would be interesting to extend the nonlinear study to the three-dimensional case (where vortex-wave interactions are known to arise at remarkably low amplitudes), as well as to higher or lower input amplitudes yielding, respectively, earlier or later onsets of nonlinearity relative to the $\sigma^{-5}$ temporal $\left(\sigma^{-4}\right.$ spatial) stage. The former amplitudes point to a nonlinear unsteady balance arising in the transient early stage, whereas the latter amplitudes are reconsidered in $\S 7.3$.

\subsection{Connections with spot experiments}

It is worth recalling the important point that the present 'spot' evolution theory is not for the turbulent case, whereas most experiments to date are. So connections between the two are probably best viewed with caution. In similar vein, only mild adverse pressure gradients are considered in the theory here.

It may be coincidental, then, that qualitative links exist with the 'spot' experiments under adverse pressure gradients by Gostelow et al. (1993, 1994, 1997), Seifert \& Wygnanski (1995), van Hest (1997) and Howell (1998). The general shape in figure $4(a)$ is not dissimilar to the observed 'spot' shapes, given the caution above. The increase in theoretical spread angle from about $11^{\circ}$ (Bowles \& Smith 1995) in the zero-gradient case to about $19^{\circ}$ in the adverse-gradient case (figure 4) is in agreement with the experimental trends (although apparently less in magnitude, see below), and likewise for the appearance of distinct new behaviour at the spanwise edge of the 
'spot', behaviour which is associated here with the (-) contributions in $\S 4$ with their longitudinal-vortex form and which is similar to that observed in the flow visualization study by Carlson et al. (1982) (see figure 3 of Johnson 1998) and in the off-centre spiking found by van Hest. The maximum disturbance amplitude nevertheless persists along the 'spot' centreline, leading there to the first internal vortex wind-up ( $\$ 5$ ).

The responses of the mean-flow alterations in $\S 6$ suggest other tentative connections, both within or under the 'spot' (figure $7 b$ ) and behind the spot trailing edge (figure $7 c, d)$. It remains to be seen whether these yield a full explanation of the calmed region in practice, although at early times, clearly, (6.6) is the dominant response, yielding the results of figure $7(b)$. Regions of significant positive mean-flow velocity are induced near the wall, in figure $7(b)$, leaving strong attachment of the flow there in view of the corresponding high mean wall shears. At increased amplitudes, or later times, the direct inertial effect of (6.6) may be connected with an empirical suggestion by Jones mentioned in $\S 1$. Simultaneously, the mean-pressure property $(6.8 a)$ may tend to cause separation after the trailing edge passes by, the mechanism then being not unlike that in the upstream separations in channel flows (Smith 1978), in roughness flows (Smith $\&$ Walton 1998) and horseshoe vortices, and being close to one suggested empirically by Seifert, with regard to the commonly observed calmed region trailing a 'spot'. That again requires study of higher amplitudes and/or increased distances travelled downstream, leading hopefully to close comparisons with Gostelow et al. and Seifert et al. on the leading-edge 'spot' velocity for various adverse pressure gradients.

We observe further that the induced mean wall shear is relatively large, dominated by the $O\left(\sigma^{19 / 2} R e^{3 / 4}\right)$ contribution to $\partial u / \partial y$ (at the wall) provoked by the slow unsteady slip $\widetilde{\tilde{u}}_{3 m}$, as opposed to the smaller $O\left(R e^{1 / 2}\right)$ contribution of the original mean flow. This is due to the associated thin $O\left(\sigma^{-5 / 2} R e^{-3 / 4}\right)$ wall layer of viscous unsteady motion which in essence starts afresh after the 'spot' has passed by, and it links, however remotely, with the experimental observations on calmed region properties by Gostelow, Hodson, Jones and Seifert. Indeed, the induced mean slip in the earlier transient stage for this case is larger, of order $\sigma^{4}$, and provokes a yet thinner $O\left(\sigma^{-1} R e^{-3 / 4}\right)$ viscous wall layer then, accompanied by larger values, of order $\sigma^{5} R e^{3 / 4}$, in $\partial u / \partial y$ at the wall.

Other features may be considered also, in a cautious manner. First, $\S \S 3$ and 4 indicate a 'spot' trailing-edge velocity equal to half that of the leading edge, a value which is not inconsistent with the above experiments. Secondly, the trends of the streamwise and normal positions of the leading and trailing edges, for instance in figure 3, are in line with van Hest's (1997) and other experiments. Thirdly, the linear amplitude growth found in $\$ \S 2-4$ and 7.4 (below) is similar to that found at the onset of an adverse pressure gradient and downstream in the maintaineddisturbance experiments of Dovgal \& Kozlov (1983, 1995), Boiko, Dovgal \& Kozlov (1989). Finally, the Benjamin-Ono-like mean effect in $\S 6$ reminds us of the Kachanov, Ryzhov \& Smith (1993) theory for zero pressure gradients, where fair agreement was found with detailed experiments. Similar detailed experiments would be helpful in the present case of adverse pressure gradients.

\subsection{Non-parallel flow effects}

Further links may rely more on incorporating non-parallel-flow effects, as the experimental 'spot' propagations continue well beyond the onset position upon which attention has been focused so far in this paper. The streamwise development of the basic flow then becomes important. (See also van Hest's comments.) There are various sources of non-parallelism, in addition to the viscous influences. 


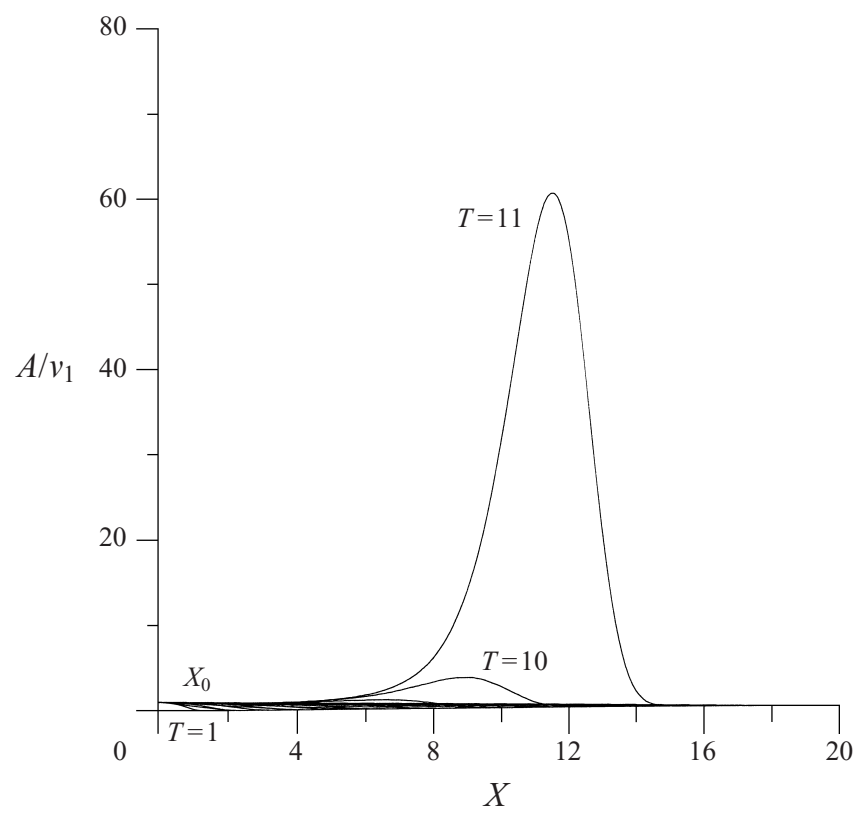

FIGURE 8. Showing non-parallel-flow effects; solution of (7.1) for $v_{1}$ small, input station at a distance $X_{0}=1$ upstream of the onset station, at times $T=1(1) 11$. Here, $\tilde{g}_{1}=\kappa=1$.

A first generalization along these lines is from $(5.12 b)$ and $(5.16 a)$ to

$$
\left(\partial_{T}+\eta \partial_{X}\right) A=\frac{1}{8} j \eta^{3}\left(\widetilde{g}_{1} X-\widetilde{g}_{1} X_{0}-3 \kappa \eta^{2}\right) A
$$

in the $X-T$ frame, with $\widetilde{g_{1}} \equiv \bar{p}^{\prime \prime}(0)$ a positive constant, $X_{0}$ an order-one constant, to account for the local linear variation of the basic pressure gradient through the pressure minimum (§2) at $X=X_{0}$, with the 'spot' being initiated at $X=0$. This applies when the slower streamwise lengthscale $O\left(R e^{-1 / 2} \sigma^{-4} X\right)$ in stage 2 becomes comparable with the $O\left(\sigma^{2}\right)$ distance $x_{1}$ from onset, i.e. when $X$ is $O\left(R e^{1 / 2} \sigma^{6}\right)$, which is large for $\sigma \gg R e^{-1 / 12}$; alternatively, $X$ then is $O(1)$ if $\sigma$ is of order $R e^{-1 / 12}$, a scale of $\sigma$ which is consistent with the earlier inviscid requirement in tier I, that $\sigma$ be greater than $O\left(R e^{-1 / 8}\right)$, and which yields a non-parallel onset lengthscale of order $R e^{-1 / 6}$. The solution of (7.1) for small disturbances where $j=\pi$, and for the same initial condition as in $\S 5.3$, gives now

$$
A=v_{1} \exp (-q \eta / 2) \exp \left[\frac{1}{8} \pi \eta^{3}\left\{\widetilde{\mathrm{g}}_{1} \frac{\eta T^{2}}{2}-\left(\widetilde{\mathrm{g}}_{1} X_{0}+3 \kappa \eta^{2}\right) T\right\}\right],
$$

when $v_{1}$ is small (see figure 8). At early times, the disturbance either decays, if $X_{0}$ is positive (corresponding to an input position upstream of the basic pressure minimum point), or grows, if $X_{0}$ is negative (for a finite range of $\eta$ values). At late times, however, exponential growth is inevitable, and, moreover, it is of the enhanced form $\exp \left[T^{6}\right]$, for an increasingly long range $0<\eta<\widetilde{g}_{1} T /(6 \kappa)$, giving spatially $X$ of order $T^{2}$. The above early- and late-time features tend to be verified by the numerical plots in figure 8 which are for the case of a disturbance input station lying upstream of the onset station, so that $X_{0}$ is positive in that case. The late-time growth is clearly very strong.

This rapid growth and lengthening of the 'spot' make good physical sense. Thus, when the 'spot' evolution time $t\left(=R e^{-1 / 2} \sigma^{-5} T\right)$ increases to $O(1)$ the total streamwise 
length $x\left(=R e^{-1 / 2} \sigma^{2} X\right)$ of the 'spot' increases to $O(1)$, from the orders of magnitude above; the associated normal distance of the leading edge from the wall $(y \sim$ $\left.R e^{-1 / 2} \sigma X /(2 T)\right)$ rises to $O\left(R e^{-1 / 2}\right)$, the boundary-layer scale; and the leading- and trailing-edge velocities both also increase, to become of $O(1)$. The growths for low input amplitudes in figures $7(b)$ and $7(c)$ also tie in with the trend. Simultaneously, the characteristic internal length and time scales of the 'spot' decrease to $O\left(R e^{-1 / 2}\right)$, from the fast dependence in (5.1).

The overall trend points to a long-scale/short-scale interaction entering next, of the kind described for zero basic pressure gradients by Smith \& Burggraf (1985), Smith et al. (1994), Li et al. (1998). For nonlinear disturbances, on the other hand, the upper limitation,

$$
A<\eta^{-1} T^{-1 / 2}
$$

still holds on the amplitude solution of (7.1), as in $\S 5$, in view of the local vortex roll-up at the 'spot' leading edge.

\subsection{Towards separation}

A second, wider, generalization is to

$$
\left(\partial_{T}+\eta \partial_{X}\right) A=\frac{1}{8} j \eta^{3}\left(\widetilde{g}_{3}(X)-3 \kappa_{3}(X) \eta^{2}\right) A,
$$

with $\widetilde{g}_{3}(X), \kappa_{3}(X)$ being given functions of $X$, concerned, in particular, with midchord breakaway separations and local blade-trailing-edge separations owing to blade thickness, incidence or camber. On the basis of triple-deck theory, in such a breakaway separation, the adverse pressure gradient sets in strongly only within an asymptotically small distance of separation (and similarly for the blade trailing-edge flows), thus allowing application there of the present onset theory. The pressure $p$ locally has the form $b_{1}\left(x_{s}-x\right)^{3 / 2}-R e^{-1 / 16} b_{2}\left(x_{s}-x\right)^{1 / 2}$, where the constants $b_{1}$ and $b_{2}$ are positive and of order unity, and $x_{s}$ is the separation point. So, the pressure minimum arises at a point $x_{\min }$ lying a distance $R e^{-1 / 16} b_{2} /\left(3 b_{1}\right)$ ahead of $x_{s}$, thus setting a relatively long streamwise lengthscale. The skin friction there is $\bar{\lambda}-O\left(\operatorname{Re}^{-1 / 16}\left(x_{s}-x\right)^{-1 / 6}\right)$ and $\widetilde{g}_{3}(X)$ in (7.4) can be deduced approximately from the local pressure function above.

More precisely, accounting for the largeness of $\bar{\tau}\left|\bar{\tau}^{\prime}\right|$ and for the pressure gradient $\bar{p}^{\prime}$ becoming positive between $x_{\min }$ and $x_{s}$ indicates three zones of interest. One is comparatively close to $x_{\min }$, where (7.1) is found to apply again over a lengthscale which is short compared with the distance $x_{s}-x_{\min }$, but slightly longer than in the non-parallel case of the previous paragraph. The second zone of interest covers the majority of the distance between $x_{\min }$ and $x_{s}$. The local maximum (over $\eta$ ) growth rate on the parallel flow basis is proportional to $\tilde{g}^{5 / 2} / \kappa^{3 / 2}$ and so increases from zero at $x_{\min }$, but it is found to have a maximum more globally over $x$, at $x_{\max }$ say, because of the factors $\tilde{g}, \kappa$ which follow from the pressure form above and from $\bar{\tau} \bar{\tau}^{\prime}$. The details show that this maximum occurs $5 / 6$ of the way from $x_{\min }$ to $x_{s}$, that is

$$
x_{\max }=x_{\min }+\frac{5}{6}\left(x_{s}-x_{\min }\right),
$$

which is relatively close to the separation point. Nonlinear effects as in $\S \S 5$ and 6 would tend to provoke breakdown prior to (7.5). Moreover, the scaled growth rate then decreases towards zero as $x$ increases further from $x_{\max }$ towards $x_{s}$. This perhaps surprising (stabilizing) feature is due to the rapid increase of $-\bar{\tau}^{\prime}$, overwhelming the increase of the effective pressure gradient $\tilde{g}$ and, indeed, eventually causing the local inflection point to migrate so close to the wall that (as expected) the inflection point 
enters the lower deck of the triple-deck structure closer to the breakaway separation position $x_{s}$.

That brings us to the third zone of interest, near $x_{s}$, where the basic pressure gradient is dominated by the $b_{2}$ term above. In consequence, the non-parallel flow equation (7.4) holds over a lengthscale of order $R e^{-3 / 14}$ near $x_{s}$, with $\tilde{g}_{3}, \kappa_{3}$ both being positive and proportional to scaled $\left(X_{s}-X\right)^{m}, m=-\frac{1}{2},-\frac{7}{6}$ in turn. The non-parallel solution then shows that a 'spot' initiated at any station within the above lengthscale ahead of $x_{s}$ produces negligible amplitude variation at $x_{s}$ itself. Again, this important conclusion is due to the surprising stabilizing feature noted just after (7.5), and it is in line with (7.5) itself.

The above seems to offer one way to understand more of the behaviour of 'spot' disturbances entering a substantial separation region. While the current work supports a view that, at an inception near a pressure minimum, all adverse pressure gradient 'spots' are essentially the same, the form of the non-parallelism (7.4) accounts for the entire length of adverse pressure gradient leading up to a mid-chord separation or to a trailing edge. Other cases or scales of non-parallelism may occur and some work on this is in progress by F. T. S., R. I. Bowles and S. N. Brown. The extensions (7.1) and (7.4) should help the theory to keep track of the 'spot' further downstream under the increasing adverse pressure gradient and may lead then to an enlarged spread angle in the three-dimensional case.

\subsection{Finally}

We end with three brief comments. First, some initial unsteady forcings are considered in the Appendix. More sustained forcing and/or interaction with free-stream turbulence should be studied further (D. Ashpis, private communications 1997), as should interactions between several 'spots'. The Appendix also considers an analogy between near-wall forcing and external forcing effects in a 'spot', with a relatively thick vortical wake outside the boundary layer providing one example of external forcing. Secondly, given the predominance of the moving critical layers in the present setting, we note that full unsteadiness may re-enter the main critical layer eventually because of vortex roll-up in the nonlinear case. This is as in the spiking theory of Li et al. (1998) and as in van Hest's (1997, pp. 139, 140) experiments: R. I. Bowles, private communications 1998. Thirdly, we would like to pursue the analysis of that nonlinear roll-up, taking place as it does at the head or leading edge of the 'spot'.

Thanks are due to Professor Paul Gostelow, Dr Avi Seifert, Dr Tom Allen, Dr Howard Hodson, Professor Terry Jones, Professor Roddam Narasimha and Dr David Ashpis for their interest, to the referees for helpful comments, and for support for F. T.S. through the Isaac Newton Institute during part of this study.

\section{Appendix A. On 'spots' from wall forcing or external forcing}

We consider here the 'spot' evolution if the initial disturbance is set up by forcing or receptivity, during the transient stage, as presented earlier in figure 1.

First, forcing at the wall is exemplified by unsteady slot suction and/or injection, e.g. a pulse, as used experimentally by Howell (1998) and others mentioned in $\S 1$ to examine the ensuing 'spot' development. Here, $(3.2 b)$ and $(3.2 c)$ are altered by the prescribed scaled normal velocity $V_{W}(\tilde{x}, \tilde{t})$ at the wall, such that now tier I has the flow solution

$$
\tilde{v}_{0}=V_{W}-\tilde{A}_{0 \tilde{x}} Y,
$$




$$
\tilde{A}_{0 \tilde{t}}+V_{W}=-\tilde{p}_{0 \tilde{x}},
$$

with the suction or injection speed being of order $\Delta \sigma$ and appearing only as above rather than in higher-order terms. In tiers II and III the induced displacement and pressure dominate the dynamics, as before, in view of the growth in $Y$ in (A 1), whereas $V_{W}(\tilde{x}, \tilde{t})$ appears only in higher-order effects there, such as an addition of $V_{W}$ to $\check{v}_{1}$ in (3.5b). Hence, (3.7) remains valid. The coupling of (A 2) and (3.7) then gives the transform equation

$$
\left(\partial_{\tilde{t}}+i k|k|\right) \tilde{A}_{0}^{*}=-V_{W}^{*}(k, \tilde{t})
$$

for $\tilde{A}_{0}^{*}(k, \tilde{t})$, which is assumed to be zero initially at time $\tilde{t}=0$, say, prior to the slot pulse $V_{W}$. Suppose for clarity that $V_{W}$ is a constant $V_{J}$ between $\tilde{x}=0, \tilde{\ell}$ (the slot), and zero otherwise, for times $\tilde{t}$ between zero and $\tilde{t}_{0}$, after which $V_{W}$ is identically zero everywhere. The solution of (A 3 ) then is

$$
\begin{gathered}
\tilde{A}_{0}^{*}=V_{J}(1-\exp (-\mathrm{i} k \tilde{\ell}))(1-\exp (-\mathrm{i} k|k| \tilde{t})) /\left(k^{2}|k|\right), \\
\tilde{A}_{0}^{*}=V_{J}(1-\exp (-\mathrm{i} k \tilde{\ell}))\left(1-\exp \left(-\mathrm{i} k|k| \tilde{t}_{0}\right)\right) \exp \left(-\mathrm{i} k|k|\left(\tilde{t}-\tilde{t}_{0}\right)\right) /\left(k^{2}|k|\right),
\end{gathered}
$$

in turn, for $0<\tilde{t}<\tilde{t}_{0}, \tilde{t}>\tilde{t}_{0}$. It follows that the effective initial condition, for the free evolution of the spot at times $\tilde{t}$ after $\tilde{t}_{0}$, is $V_{J}$ multiplied by the first two bracketed terms in (A 5) divided by $k^{2}|k|$, imposed at $\tilde{t}=\tilde{t}_{0}$. This replaces $R^{*}(k)$ in (3.8) and subsequently (we note $R^{*}(0)$ is finite), with $\tilde{t}-\tilde{t}_{0}$ replacing $\tilde{t}$, and so the spot goes on to develop just as described throughout $\S \S 3-6$.

Here and below, the resulting disturbance such as in (A 5) or (3.8) does not exhibit on its own any sign of growth or decay during the current transient stage 1, of course, and little indication is given by the higher-order term $\tilde{A}_{3}$. Instead, growth or decay is seen fully only in the subsequent stage 2 . So, if the narrow injection slot implied above for (A 4) and (A 5) is positioned downstream of the adverse-pressure-gradient onset station $x=0$, so that $x_{1}>0$, then spot growth results directly during stage 2 as in $\S \S 5$ and 6. If the slot position is upstream, so that $x_{1}<0$, then the spot decays at first but grows later, downstream, as the non-parallel-flow effects come into action as described in $\S 7.3$ and shown in figure 8. The intermediate case of slot positioning closer to the onset station yields slower growth or decay, in line with $\S 7.3$.

Secondly, a forcing which has been much studied previously, e.g. Terent'ev (1981), Hall \& Smith (1982), Goldstein (1985), is that due to a combination of temporal oscillation in the free stream and a bump at the wall, or other source of near-surface non-parallelism. This seems less relevant here, however, unless rough surfaces, for example, are under consideration.

Thirdly, forcing due solely to perturbed conditions in the free stream external to the boundary layer may take numerous forms. Suppose the input emanates from a disturbance outside the upper tier III of (3.6), for instance from unsteadiness in a remote upstream incident wake which is typically thicker than the wall boundary layer. The outside disturbance then acts as a forcing term via the outer boundary condition on the linearized Euler equations in the upper tier, such as in one of the forms

$$
\begin{gathered}
\hat{p}_{0} \sim \pi_{1}(\tilde{t}) \exp \left(\alpha_{1} \overline{\bar{y}}\right) \cos \left(\alpha_{1} \tilde{x}-\omega_{1}(\tilde{t})\right), \\
\hat{p}_{0} \sim \pi_{2}(\tilde{t}) r^{m_{2}} \cos \left(m_{2} \theta+m_{3}\right), \\
{\left[\hat{u}_{0}, \hat{v}_{0}\right] \sim\left[\cos \alpha_{3}, \sin \alpha_{3}\right] \pi_{3}(\tilde{t}) f_{3}\left(\tilde{x} \sin \alpha_{3}-\overline{\bar{y}} \cos \alpha_{3}-\omega_{3}(\tilde{t})\right),}
\end{gathered}
$$

as $r \equiv\left(\tilde{x}^{2}+\overline{\bar{y}}^{2}\right)^{1 / 2} \rightarrow \infty$. Here, $\tan \theta=\overline{\bar{y}} / \tilde{x}$, while $\pi_{1}, \pi_{2}, \pi_{3}, \alpha_{1}, \omega_{1}, m_{2}, m_{3}, \alpha_{3}, \omega_{3}$ are 
prescribed $O(1)$ functions of time $\tilde{t}$ and the given wake-profile function $f_{3}$ is also $O(1)$, typically. The constraints (A 6) and (A 8) can arise from remote wake disturbances, as in Smith et al.'s (2000) $§ 5$ and 6, respectively. For the former case, the shear in the remote wake is much less than in the present wall boundary layer because the wake is thicker, while strictly its minimum velocity is small, yielding the convective contribution $\omega_{1}$; the flow solutions in Smith et al. have $\pi_{1}(\tilde{t})$ being oscillatory in $\tilde{t}$, along with exponential dependence in the normal direction if the wake perturbations are simple waves. The constraint (A 7) is in similar vein, again consistent with irrotational motion in tier III.

The condition (A 8) is more clearly equivalent to a distorted wake profile (its evolution defined by $\left.\pi_{3}, f_{3}, \alpha_{3}\right)$ with vorticity incident at an $O(1)$ angle $\alpha_{3}(\tilde{t})$ and with zero incoming pressure. It is also in keeping with the Smith et al. near-wake disturbance theory for general perturbations rather than the simple waves of (A 6), again in the remote thick wake directed at an angle to the wall. The high degree of arbitrariness possible through $\pi_{3}, f_{3}, \alpha_{3}$ in this external wake is due to the local outer flow being quasi-steady, as well as nearly uniform. Again, see figure 1. Taking (A 8), we obtain from the linearized governing equations in tier III the interaction response, as the wall is approached,

$$
\tilde{p}_{0}(\tilde{x}, \tilde{t})=\frac{1}{\pi}(P . V .) \int_{-\infty}^{\infty}\left(\tilde{A}_{0 \xi}-\tilde{G}_{0}\right) \frac{\mathrm{d} \xi}{(\tilde{x}-\xi)}
$$

in place of (3.7) now, with suitable boundedness in the far field. Here, $\tilde{G}_{0}(\tilde{x}, \tilde{t})$ denotes $\pi_{3} \sin ^{2}\left(\alpha_{3}\right) f_{3}\left(\tilde{x} \sin \alpha_{3}-\omega_{3}\right)$. The response (A 9) is coupled with $(3.2 c)(n=0)$, to determine the induced pressure and displacement due to the external forcing. The unknown difference function $\tilde{A}_{0}-\tilde{g}_{0}$, where $\partial \tilde{g}_{0} / \partial \tilde{x}=\tilde{G}_{0}$, therefore satisfies (3.7) coupled with (A2) again, for an effective injection velocity which is $V_{W}=\partial \tilde{g}_{0} / \partial \tilde{t}$, or

$$
V_{W}(\tilde{x}, \tilde{t})=\frac{\partial}{\partial \tilde{t}}\left\{\pi_{3}\left(\sin \alpha_{3}\right) g_{3}\left(\tilde{x} \sin \alpha_{3}-\omega_{3}\right)\right\} .
$$

Here, $g_{3}$ is the incident mass-flux-deficit profile associated with (A 8), so that $\hat{\psi}_{0} \sim$ $-\pi_{3} g_{3}(s)$ there, $s=\tilde{x} \sin \alpha_{3}-\overline{\bar{y}} \cos \alpha_{3}-\omega_{3}$, and $g_{3}^{\prime}=f_{3}$. The equivalent injection/suction velocity at the wall therefore relies on the incident external-wake-like amplitude $\pi_{3}$, angle $\alpha_{3}$ and/or speed factor $\omega_{3}$ being unsteady, together with $\alpha_{3}$ being non-zero.

Let us continue further with the analogy. Suppose, for clarity again, that the speed factor $\omega_{3}$ is simply $\tilde{\omega} \tilde{t}$, with $\tilde{\omega}, \tilde{\pi}_{3}, \alpha_{3}$ constant, leaving

$$
V_{W}(\tilde{x}, \tilde{t})=-\tilde{\omega} \pi_{3}\left(\sin \alpha_{3}\right) f_{3}\left(\tilde{x} \sin \alpha_{3}-\tilde{\omega} \tilde{t}\right) .
$$

If, also, the incident velocity profile $f_{3}(s)$ is piecewise constant, $\pm \tilde{f}$, so that $g_{3}(s)$ is piecewise linear but with no added mass in total, only momentum, then (A11) gives a blow-suck piecewise constant $V_{W} \propto \pm \tilde{f}$ over the ranges $0<\tilde{x}-\tilde{c} \tilde{t}<\tilde{\ell}$, $\tilde{\ell}<\tilde{x}-\tilde{c} \tilde{t}<2 \tilde{\ell}$, in turn, for all positive $\tilde{t}$, say. The effective scaled speed of the (moving) slot position is $\tilde{c}=\tilde{\omega} /\left(\sin \alpha_{3}\right)$ and $\tilde{\ell}$ is again a lengthscale.

Hence (compare (A 3 ) and (A 4))

$$
\tilde{A}_{0}^{*}=\tilde{g}_{0}^{*}+\frac{V_{J}}{|k|-\tilde{c}}\left(\frac{1-\mathrm{e}^{-\mathrm{i} k \tilde{t}}}{\mathrm{i} k}\right)^{2}\left(\mathrm{e}^{-\mathrm{i} k|k| \tilde{t}}-\mathrm{e}^{-\mathrm{i} k \tilde{c} \tilde{t}}\right),
$$

where now the constant $V_{J}=-\tilde{\omega} \pi_{3}\left(\sin \alpha_{3}\right) \tilde{f}$. Of particular concern is the case where the external speed factor $\tilde{\omega}$ is relatively large. This makes $\tilde{c}$ large and so $|k|$ may be neglected (except at very large values) in the denominator of (A 12). Then the 
$\exp (-\mathrm{i} k \tilde{c} \tilde{t})$ term and the $\tilde{g}_{0}^{*}$ contribution represent the rapid part of the disturbance that travels downstream with the forcing, at high relative speed $\tilde{\omega} / \sin \alpha_{3}$, while the $\exp (-\mathrm{i} k|k| \tilde{t})$ term represents the part of the disturbance that is left behind, at slower speed. The latter part, namely

$$
\tilde{A}_{0}^{*}=\pi_{3}\left(\sin ^{2} \alpha_{3}\right)\left(\frac{1-\mathrm{e}^{-\mathrm{i} k \tilde{\ell}}}{\mathrm{i} k}\right)^{2} \tilde{f} \mathrm{e}^{-\mathrm{i} k|k| \tilde{t}}
$$

goes on to form the 'spot', cf. (3.8) and (3.10). Here, $R^{*}(0)=\pi_{3}\left(\sin ^{2} \alpha_{3}\right) \tilde{f} \tilde{\ell}^{2}$ is again finite; notably, it (and (A 13)) is independent of $\tilde{\omega}$ owing to a cancellation above, leaving the subsequent 'spot' properties overall controlled by the incident amplitude, angle and profile width alone.

Further, taking (A 6) and (A 7) likewise provokes virtually the same forced equation as in (A 2), but with the effective $V_{W}$ not necessarily having compact support and so, then, leaving $R^{*}(k)$ unbounded at small $|k|$. This affects the amplitude scales in stage 2, although less so the main dynamics. Also, these more spatially extensive disturbances point once more to the non-parallel decay-growth evolution of $\S 7.3$ upstream/downstream of the adverse-onset station, as does the fast-moving slot analogy of (A 10)-(A 13) if it enters far upstream.

Other external or near-wall forcings are produced by a moving body in the free stream or by a travelling patch of vorticity and basic non-parallelism near the wall, for instance. Their equivalent suction/injection wall velocities could be of interest. Finally, the three-dimensional counterpart $(\S 4)$ also holds, based on a suction/injection hole of circular cross-section, for example.

\section{REFERENCES}

Abu Ghannam, B. J. \& Shaw, R. 1980 Natural transition of boundary layers - the effects of turbulence pressure gradient and flow history. J. Mech. Engng Sci. 22, 213-228.

BoIKo, A. V., Dovgal, A. V. \& Kozlov, V. V. 1989 Nonlinear interactions between perturbations in transition to turbulence in the zone of laminar boundary layer separation. Sov. J. Appl. Phys. 3, 46-52.

Bowles, R. I. 2000 Transition to turbulent flow in aerodynamics. Phil. Trans. R. Soc. A 358, 245-260.

Bowles, R. G. A. \& Smith, F. T. 1995 Short-scale effects on model boundary-layer spots. J. Fluid Mech. 295, 395-407.

BRown, S. N. \& SMith, F. T. 1999 Spot concentrations of large-amplitude disturbances in laminar boundary layers. Q. J. Mech. Appl. Maths 52, 269-281.

Carlson, D. R., Widnall, S. E. \& Peeters, M. F. 1982 A flow-visualization study of transition in plane Poiseulle flow. J. Fluid Mech. 121, 487-505.

Chapman, C. J. \& Proctor, M. R. E. 1980 Nonlinear Rayleigh-Bénard convection between poorly conducting boundaries. J. Fluid Mech. 101, 759-782.

Clarke, J. P., Jones, T. V. \& LaGrafF, J. E. 1994 On the propagation of naturally-occurring turbulent spots. J. Engng Maths 28, 1-19.

Dovgal, A. V. \& Kozlov, V. V. 1983 Influence of acoustic perturbations on the flow structure in a boundary layer with adverse pressure gradient. Fluid Dyn. 18, 205-209.

Dovgal, A. V. \& Kozlov, V. V. 1995 On nonlinearity of transitional boundary-layer flows. Phil. Trans. R. Soc. A 352, 473-482.

GaJjar, J. S. B. \& Smith, F. T. 1985 On the global instability of free disturbances with a timedependent nonlinear viscous critical layer. J. Fluid Mech. 157, 53-77.

GoldsteIN, M. E. 1985 Scattering of acoustic waves into Tollmien-Schlichting waves by small streamwise variations in surface geometry. J. Fluid Mech. 154, 509-529.

Goldstein, M. E., Durbin, P. A. \& LeIB, S. J. 1987 Roll-up of vorticity in adverse-pressure-gradient boundary layers. J. Fluid Mech. 183, 325-342. 
Gostelow, J. P., Blunden, A. W. \& Walker, G. J. 1994 Effects of free-stream turbulence and adverse pressure gradients on boundary-layer transition. ASME J. Turbomachinery 116, 392-404.

Gostelow, J. P., Hong, G., Melwani, N. \& Walker, G. J. 1993 Turbulent spot development under a moderate pressure gradient. ASME paper 93-GT-377.

Gostelow, J. P., Walker, G. J., Solomon, W. J., Hong, G. \& Melwani, N. 1997 Investigation of the calmed region behind a turbulent spot. ASME J. Turbomachinery 119, 802-809.

Hall, P. \& Sмiтh, F. T. 1982 A suggested mechanism for nonlinear wall roughness effects on high Reynolds number flow stability. Stud. Appl. Maths 66, 241-265.

Hest, B. F. A. van $1997 \mathrm{PhD}$ thesis, Technical University Delft.

Hickernell, F. J. 1984 Time-dependent critical layers in shear flows on the beta-plane. J. Fluid Mech. 142, 431-449.

Howell, R. J. 1998 PhD thesis, University of Cambridge.

Jennings, M. J., Stewart, P. A. \& Cowley, S. J. 1999 When is the weakly nonlinear evolution of a localized disturbance governed by the Ginzburg-Landau equation? Proc. R. Soc. A 455, $1521-1560$.

Johnson, M. W. 1997 Proc. workshop on boundary layer transition in turbomachinery, Minnowbrook II, Syracuse, New York, September 1997.

JoHnson, M. W. 1998 Turbulent spot characteristics in boundary layers subjected to streamwise pressure gradient. ASME paper 98-GT-124, pp. 1-8, presented at Intl Gas Turbine \& Aeroengine Congr. Stockholm, 2-5 June 1998.

Johnson, M. W. \& ErCAN, A. H. 1996 A boundary layer transition model. ASME paper 96-GT-444.

Kachanov, Y. S., Ryzhov, O. S. \& Sмith, F. T. 1993 Formation of solitons in transitional boundary layers: theory and experiment. J. Fluid Mech. 251, 273-297.

Li, L., Walker, J. D. A., Bowles, R. I. \& Smith, F. T. 1998 Short-scale break-up in unsteady interactive layers: local development of normal pressure gradients and vortex wind-up. J. Fluid Mech. 374, 335-378.

Medeiros, M. A. F. \& Gaster, M. 1996 The nonlinear behaviour of modulated Tollmien-Schlichting waves, IUTAM Symp. on Nonlinear Instability and Transition in Three-Dimensional Boundary Layers (ed. P. W. Duck \& P. Hall), pp. 197-206. Kluwer.

NARAsimha, R. 1997 Report of workshop on boundary layer transition in turbomachines, Minnowbrook II, Syracuse, New York, NASA/CP-1998-206958, September 1997.

Savin, D. J., Smith, F. T. \& Allen, T. 1999 Transition of free disturbances in inflectional flow over an isolated surface roughness. Proc. R. Soc. A 455, 491-541.

SEIFERT, A. 1997 Proc. workshop on boundary layer transition in turbomachinery, Minnowbrook II, Syracuse, New York, September 1997.

Seifert, A. \& Hodson, H. P. 2000 Periodic turbulent strips and calmed regions in a transitional boundary layer. AIAA tech. note, submitted.

Seifert, A. \& Wygnanski, I. 1995 On turbulent spots in a laminar boundary layer subjected to a self-similar adverse pressure gradient. J. Fluid Mech. 296, 185-209.

Smith, F. T. 1978 Flow through symmetrically constricted tubes. J. Inst. Maths Applics 21, 145-156.

Sмiтн, F. T. 1993 Theoretical aspects of transition and turbulence in boundary layers. AIAA J. 31, $2220-2226$.

Smith, F. T. 1995 On spikes and spots. Phil. Trans. R. Soc. A 352, 405-424.

Smith, F. T. \& Bodonyi, R. J. 1981 The upper-branch stability of the Blasius boundary layer, including nonparallel flow effects. Proc. R. Soc. A 375, 65-92.

Smith, F. T. \& Bodonyi, R. J. 1987 Properties of a time-dependent nonlinear viscous critical layer. Stud. Appl. Maths 77, 129-150.

Smith, F. T. \& Bowles, R. I. 1992 Transition theory and experimental comparisons on (I) amplification into streets and (II) a strongly nonlinear breakup criterion. Proc. R. Soc. A 439, $163-175$.

Smith, F. T., Bowles, R. G. A. \& Li, L. 2000 Nonlinear effects in absolute and convective instabilities of a near-wake. Europ. J. Mechs B 19, 173-211.

Smith, F. T. \& BURGGraF, O. R. 1985 On the development of large-sized short-scaled disturbances in boundary layers. Proc. R. Soc. A 399, 25-55.

Smith, F. T., Dodia, B. T, Bowles, R. G. A. 1994 On global and internal dynamics of spots: a theoretical approach. J. Engng Maths 28, 73-91. 
Smith, F. T. \& Doorly, D. J. 1992 Initial-value problems for spot disturbances in incompressible or compressible boundary layers. J. Engng Maths 26, 87-106.

Smith, F. T. \& Walton, A. G. 1998 Flow past a two- or three-dimensional steep-edged roughness. Proc. R. Soc. A 454, 31-69.

Solomon, W. J., Walker, G. J. \& Gostelow, J. P. 1996 Transition length prediction for flows with rapidly changing pressure gradients. ASME J. Turbomachinery, 118, 744-751.

Steelant, J. \& Dick, E. 1996 Calculation of transition in adverse pressure gradient flow by conditioned equations. Trans. ASME 96-GT-160, presented Intl Gas Turbine \& Aeroengng Congr. Birmingham, UK, 10-13 June 1996.

Stewartson, K. \& Stuart, J. T. 1971 A nonlinear instability theory for a wave system in plane Poiseuille flow. J. Fluid Mech. 48, 529-545.

TERENT'EV, E. D. 1981 Linear problem for a vibrator in subsonic boundary layer. Prikl. Math. Mech. 45, 1049-1055.

Whitham, G. B. 1974 Linear and Nonlinear Waves. Wiley, New York.

Zhuk, V. I. \& Ryzhov, O. S. 1982 Locally inviscid perturbations in a boundary layer with selfinduced pressure. Dokl. Akad. Nauk SSSR 263, 56-59 (English Transl. in Sov. Phys. Dokl. 27, $177-179,1982)$ 